\title{
Geodesic motion of S2 and G2 as a test of the fermionic dark matter nature of our Galactic core
}

\author{
E. A. Becerra-Vergara ${ }^{1,2,3}$, C. R. Argüelles ${ }^{1,2,4}$, A. Krut ${ }^{1,2}$, J. A. Rueda ${ }^{1,2,5,6,7}$, and R. Ruffini ${ }^{1,2,5,6,8}$ \\ 1 ICRANet, Piazza della Repubblica 10, 65122 Pescara, Italy \\ e-mail: eduar . becerra@icranet.org \\ 2 ICRA, Dipartimento di Fisica, Sapienza Università di Roma, P.le Aldo Moro 5, 00185 Rome, Italy \\ e-mail: jorge.rueda@icra.it, ruffini@icra.it \\ ${ }^{3}$ Grupo de Investigación en Relatividad y Gravitación, Escuela de Física, Universidad Industrial de Santander, A. A. 678, \\ Bucaramanga 680002, Colombia \\ ${ }^{4}$ Facultad de Ciencias Astronómicas y Geofísicas, Universidad Nacional de La Plata, Paseo del Bosque, B1900FWA La Plata, \\ Argentina \\ 5 ICRANet-Ferrara, Dipartimento di Fisica e Scienze della Terra, Università degli Studi di Ferrara, Via Saragat 1, 44122 Ferrara, \\ Italy \\ 6 Dipartimento di Fisica e Scienze della Terra, Università degli Studi di Ferrara, Via Saragat 1, 44122 Ferrara, Italy \\ 7 INAF, Istituto de Astrofisica e Planetologia Spaziali, Via Fosso del Cavaliere 100, 00133 Rome, Italy \\ ${ }^{8}$ INAF, Viale del Parco Mellini 84, 00136 Rome, Italy
}

Received 30 May 2019 / Accepted 22 July 2020

\begin{abstract}
The motion of S-stars around the Galactic center implies that the central gravitational potential is dominated by a compact source, Sagittarius A* (Sgr A*), which has a mass of about $4 \times 10^{6} M_{\odot}$ and is traditionally assumed to be a massive black hole (BH). The explanation of the multiyear accurate astrometric data of the S2 star around Sgr A*, including the relativistic redshift that has recently been verified, is particularly important for this hypothesis and for any alternative model. Another relevant object is G2, whose most recent observational data challenge the scenario of a massive $\mathrm{BH}$ : its post-pericenter radial velocity is lower than expected from a Keplerian orbit around the putative massive $\mathrm{BH}$. This scenario has traditionally been reconciled by introducing a drag force on G2 by an accretion flow. As an alternative to the central BH scenario, we here demonstrate that the observed motion of both S2 and G2 is explained in terms of the dense core - diluted halo fermionic dark matter (DM) profile, obtained from the fully relativistic RuffiniArgüelles-Rueda (RAR) model. It has previously been shown that for fermion masses $48-345 \mathrm{keV}$, the RAR-DM profile accurately fits the rotation curves of the Milky Way halo. We here show that the solely gravitational potential of such a DM profile for a fermion mass of $56 \mathrm{keV}$ explains (1) all the available time-dependent data of the position (orbit) and line-of-sight radial velocity (redshift function $z$ ) of S2, (2) the combination of the special and general relativistic redshift measured for S2, (3) the currently available data on the orbit and $z$ of G2, and (4) its post-pericenter passage deceleration without introducing a drag force. For both objects, we find that the RAR model fits the data better than the BH scenario: the mean of reduced chi-squares of the time-dependent orbit and $z$ data are $\left\langle\bar{\chi}^{2}\right\rangle_{\mathrm{S} 2, \mathrm{RAR}} \approx 3.1$ and $\left\langle\bar{\chi}^{2}\right\rangle_{\mathrm{S} 2, \mathrm{BH}} \approx 3.3$ for $\mathrm{S} 2$ and $\left\langle\bar{\chi}^{2}\right\rangle_{\mathrm{G} 2, \mathrm{RAR}} \approx 20$ and $\left\langle\bar{\chi}^{2}\right\rangle_{\mathrm{G} 2, \mathrm{BH}} \approx 41$ for $\mathrm{G} 2$. The fit of the corresponding $z$ data shows that while for S2 we find comparable fits, that is, $\bar{\chi}_{z, \mathrm{RAR}}^{2} \approx 1.28$ and $\bar{\chi}_{z, \mathrm{BH}}^{2} \approx 1.04$, for $\mathrm{G} 2$ the RAR model alone can produce an excellent fit of the data, that is, $\bar{\chi}_{z, \mathrm{RAR}}^{2} \approx 1.0$ and $\bar{\chi}_{z, \mathrm{BH}}^{2} \approx 26$. In addition, the critical mass for gravitational collapse of a degenerate $56 \mathrm{keV}$-fermion DM core into a $\mathrm{BH}$ is $\sim 10^{8} M_{\odot}$. This result may provide the initial seed for the formation of the observed central supermassive $\mathrm{BH}$ in active galaxies, such as M 87.
\end{abstract}

Key words. Galaxy: center - Galaxy: kinematics and dynamics - Galaxy: structure - dark matter - elementary particles

\section{Introduction}

The monitoring of the motion of the so-called S-stars near the Galactic center over the past decades has revealed that the gravitational potential in which they move is dominated by a massive compact source at the center, Sagittarius A* (Sgr A*; Gillessen et al. 2009, 2017). The S-star dynamics implies a mass for Sgr $A^{*}$ of $\approx 4.1 \times 10^{6} M_{\odot}$, which is traditionally associated in the literature with a massive black hole $(\mathrm{BH}$; Gravity Collaboration 2018a; Ghez et al. 2008; Genzel et al. 2010).

Of the objects that move near and around Sgr A*, S2 and G2 are the most interesting. The star S2 describes an elliptical orbit that is focused on Sgr $\mathrm{A}^{*}$ and has a period of $16.05 \mathrm{yr}$ and the second closest pericenter of the S-stars, $r_{p(S 2)} \approx 0.6 \mathrm{mpc}$ (Gillessen et al. 2009, 2017). The S2 orbit constrains the Sgr A* mass best, but its pericenter at $\sim 1500 r_{\mathrm{Sch}}$ from $\mathrm{Sgr} \mathrm{A}^{*}$ is too far to univocally infer a putative massive $\mathrm{BH}$ of Schwarzschild radius $r_{\mathrm{Sch}}=2 G M_{\mathrm{BH}} / c^{2}$, where $M_{\mathrm{BH}}$ is its mass.

The most recent measurements of the motion of $\mathrm{G} 2$ after the peripassage around Sgr A* represent a further challenge for the hypothesis of a massive $\mathrm{BH}$. The $\mathrm{G} 2$ radial velocity is lower than that from a Keplerian motion around the massive $\mathrm{BH}$, which has been reconciled by introducing the action of a drag force exerted by an accretion flow (Plewa et al. 2017; Gillessen et al. 2019).

Our aim here is to show that the dense core - diluted halo DM density distribution of a general relativistic system of $56 \mathrm{keV}$ fermions, following the extended Ruffini-ArgüiellesRueda (RAR) model (Argüelles et al. 2018, 2019a) instead explains the orbits of S2 and G2 without invoking the massive $\mathrm{BH}$ or a drag force. We use the most complete data of 
the S2 orbit over the last 26 yr (Gillessen et al. 2017; Gravity Collaboration 2018b), including the recent data released by Do et al. (2019), and the four-year data of the G2 motion after its pericenter passage (Gillessen et al. 2019).

\section{Ruffini-Argüelles-Rueda model of dark matter}

The Ruffini-Argüelles-Rueda (RAR) model equilibrium equations consist of the Einstein equations in spherical symmetry for a perfect fluid energy-momentum tensor. Pressure and density are given by Fermi-Dirac statistics, and the closure relations are determined by the Klein and Tolman conditions of thermodynamic equilibrium (Ruffini et al. 2015). The solution to this system of equations leads to a continuous and novel dense core - diluted halo DM profile from the center all the way to the galactic halo (see Siutsou et al. 2015; Argüelles et al. 2016; Mavromatos et al. 2017, for its applications). Similar core-halo profiles with applications to fermionic DM were also obtained in Bilic et al. (2002) and more recently in Chavanis et al. (2015) from a statistical approach within Newtonian gravity.

The above corresponds to the original version of the RAR model, with a unique family of density profile solutions that behaves as $\rho(r) \propto r^{-2}$ at large radial distances from the center. This treatment was extended in Argüelles et al. (2018), by introducing a cutoff in momentum space in the distribution function (DF; i.e., accounting for particle-escape effects) that allows defining the galaxy border (see Appendix A). This extension of the RAR model was successfully applied to explain the Milky Way rotation curve, as shown in Fig. 1, implying a more general dense core - diluted halo behavior for the DM distribution as follows:

- A DM core with radius $r_{\mathrm{c}}$ (defined at the first maximum of the twice-peaked rotation curve), whose value is shown to be inversely proportional to the particle mass $m$, in which the density is nearly uniform. This central core is supported against gravity by the fermion degeneracy pressure, and general relativistic effects are appreciable.

- Then, there is an intermediate region characterized by a sharply decreasing density where quantum corrections are still important, followed by an extended and diluted plateau. This region extends until the halo scale-length $r_{\mathrm{h}}$ is achieved (defined at the second maximum of the rotation curve).

- Finally, the DM density reaches a Boltzmann regime supported by thermal pressure with negligible general relativistic effects, and shows a behavior $\rho \propto r^{-n}$ with $n>2$ that is due to the phase-space distribution cutof. This leads to a DM halo bounded in radius (i.e., $\rho \approx 0$ occurs when the particle escape energy approaches zero).

As was explicitly shown in Argüelles et al. (2019b,a, 2018), this type of dense core - diluted halo density profile suggests that the DM might explain the mass of the dark compact object in Sgr A* as well as the halo mass. It applies not only to the Milky Way, but also to other galactic structures from dwarfs and ellipticals to galaxy clusters (Argüelles et al. 2019a). Specifically, a Milky Way analysis (Argüelles et al. 2018) has shown that this DM profile can indeed explain the dynamics of the closest S-cluster stars (including S2) around Sgr A*, all the way to the halo rotation curve without changing the baryonic bulge-disk components. The analysis of the S-stars was made through a simplified circular velocity analysis in general relativity, constraining the allowed fermion mass to $m c^{2} \approx 50-345 \mathrm{keV}$. We extend this analysis by fully reconstructing the geodesic of the object in full general relativity, and apply it to S2 and G2. Figure 1 shows

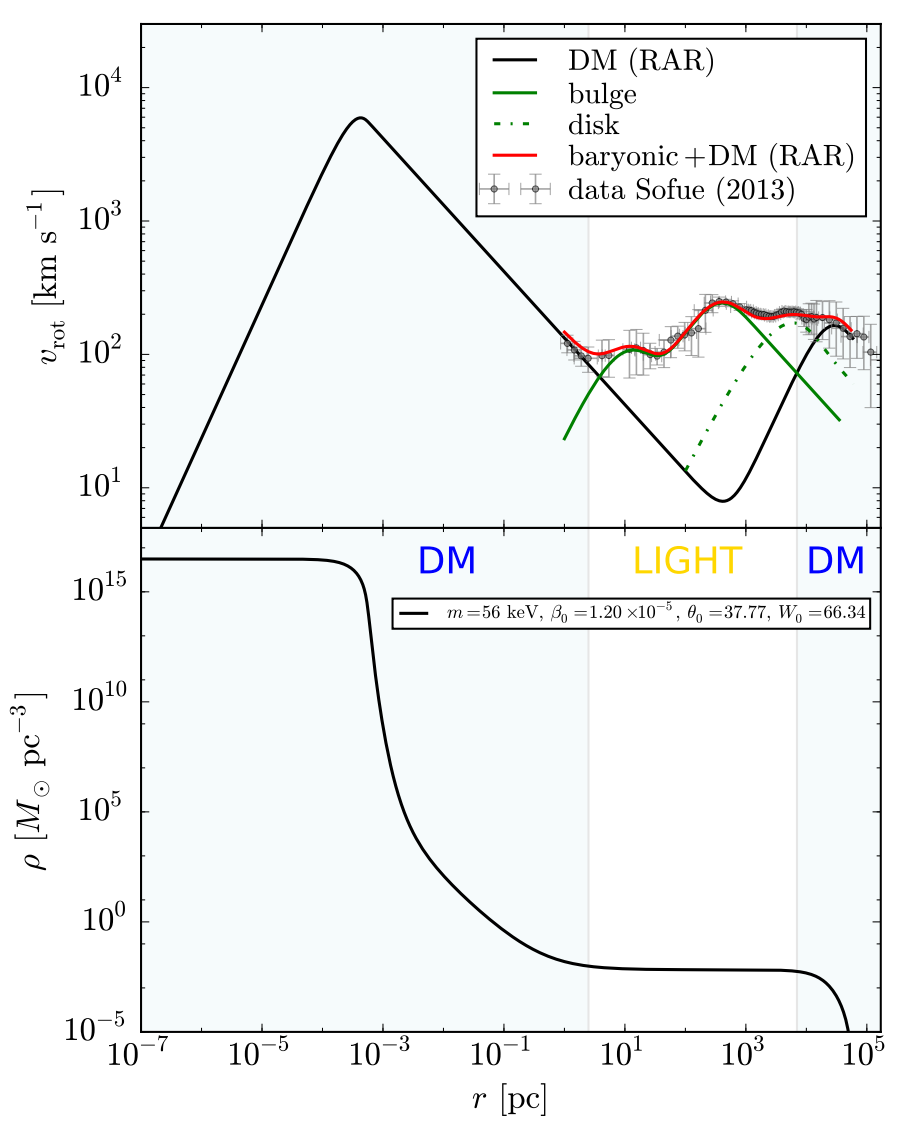

Fig. 1. Milky Way rotation curve and DM density profile from the extended RAR model with a core mass of $M_{\mathrm{c}}=M\left(r_{\mathrm{c}}\right)=3.5 \times 10^{6} M_{\odot}$. Top: DM (black) and baryonic (bulge + disk) contribution to the rotation curve $v_{\text {rot }}$ (total in red). Bottom: DM density profile. The baryonic model and the data are taken from Sofue (2013). The parameters of the extended RAR model in this case are fermion mass $m c^{2}=56 \mathrm{keV}$, temperature parameter $\beta_{0}=1.1977 \times 10^{-5}$, degeneracy parameter $\theta_{0}=37.7656$, and energy cutoff parameter $W_{0}=66.3407$. For the RAR model fitting of the Milky Way, we follow Argüelles et al. (2018); see also Appendix A.

the DM density profile and its contribution to the rotation curve for the Milky Way for $56 \mathrm{keV}$ DM fermions.

\section{Orbit and radial velocity of $\mathbf{S 2}$ and $\mathrm{G} 2$}

To obtain the S2 or G2 positions (orbit) and the corresponding line-of-sight radial velocity (i.e., the redshift function; see Appendix B) at each time, we solved the equations of motion for a test particle (see Appendix C) in the gravitational field produced by two possible scenarii that we describe below.

1. A central Schwarzschild massive BH. Gravity Collaboration (2018b) reported a $\mathrm{BH}$ mass of $M_{\mathrm{BH}}=$ $4.1 \times 10^{6} M_{\odot}$ from the fit of the most recent measurements of the position and velocity of $\mathrm{S} 2$. The more recent analysis by Do et al. (2019) reported a BH mass of $3.975 \times 10^{6} M_{\odot}$. These works used a second-order post-Newtonian (2PN) model to describe the object motion. In order to compare and contrast the $\mathrm{BH}$ and the DM-RAR hypotheses on the same ground, that is, using the same analysis method and treatment, we performed our own fit of the data for the $\mathrm{BH}$ case using a full general relativistic modeling by solving the equations of motion in the Schwarzschild metric (see Appendix C). From our analysis of $\mathrm{S} 2$, we obtain model parameters that are very similar (but not 
Table 1. Summary of the inferred best-fit values of the model and the (osculating) orbital parameters for S2 and G2 within the RAR model (fermion mass $56 \mathrm{keV}$, DM core mass $M_{\mathrm{c}}=3.5 \times 10^{6} M_{\odot}$ ) and the massive $\mathrm{BH}$ model $\left(\mathrm{BH}\right.$ mass $M_{\mathrm{BH}}=4.075 \times 10^{6} M_{\odot}$ ).

\begin{tabular}{lccccc}
\hline \hline \multirow{2}{*}{ Parameter } & \multicolumn{2}{c}{$\mathrm{S} 2$} & & \multicolumn{2}{c}{$\mathrm{G} 2$} \\
\cline { 2 - 3 } \cline { 6 - 6 } & $\mathrm{RAR}$ & $\mathrm{BH}$ & & $\mathrm{RAR}$ & $\mathrm{BH}$ \\
\hline Semimajor axis, $a(\mathrm{as})$ & 0.1252 & 0.1252 & & 1.0960 & 1.1941 \\
Eccentricity, $e$ & 0.8866 & 0.8863 & & 0.9823 & 0.9853 \\
Distance to pericenter, $r_{\mathrm{p}}(\mathrm{as})$ & 0.0142 & 0.0143 & & 0.0194 & 0.0180 \\
Distance to apocenter, $r_{\mathrm{a}}(\mathrm{as})$ & 0.2361 & 0.2362 & & 2.1725 & 2.3701 \\
Argument of pericenter, $\omega\left(^{\circ}\right)$ & 66.7724 & 66.4697 & & 81.8391 & 82.0001 \\
Inclination, $i\left(^{\circ}\right)$ & 134.3533 & 134.3505 & & 121.8993 & 119.1000 \\
Ascending node, $\Omega\left({ }^{\circ}\right)$ & 228.0240 & 227.9681 & & 50.8398 & 50.7782 \\
$X_{0}$ (mas) & -0.1557 & -0.0830 & & 0.0248 & 0.0251 \\
$Y_{0}$ (mas) & 2.5527 & 2.4893 & & -0.0160 & -0.0140 \\
Orbital period, $P(\mathrm{yr})$ & 16.0539 & 16.0506 & & 416.3400 & 470.1610 \\
\hline $\bar{\chi}_{X}^{2}$ & 1.5964 & 1.8004 & & 33.3339 & 83.9950 \\
$\bar{\chi}_{Y}^{2}$ & 6.3411 & 7.2332 & & 26.8419 & 11.2646 \\
$\bar{\chi}_{z}^{2}$ & 1.2799 & 1.0421 & & 0.9960 & 26.3927 \\
$\left\langle\bar{\chi}^{2}\right\rangle$ & 3.0725 & 3.3586 & & 20.3906 & 40.5507 \\
\hline
\end{tabular}

Notes. We refer to Appendix $\mathrm{C}$ for details of the definition of the parameters and the fitting procedure.

equal) to those that were presented in Gravity Collaboration (2018b) and Do et al. (2019); see Table 1. In particular, we obtain a BH mass of $M_{\mathrm{BH}}=4.075 \times 10^{6} M_{\odot}$.

2. A fermionic DM distribution obtained from the extendedRAR model; see Appendix A. As was shown in Argüelles et al. (2018), the fermion mass must be higher than $48 \mathrm{keV}$ and lower than $345 \mathrm{keV}$. We here present the results of the solution of the equations of motion in the metric produced by the DM distribution of $56 \mathrm{keV}$ fermions, with corresponding RAR model parameters as shown in Fig. 1. We obtain an excellent fit of the data for a mass of the DM quantum-core, $M_{\mathrm{c}} \equiv M\left(r_{\mathrm{c}}\right)=3.5 \times 10^{6} M_{\odot}$; see Table 1.

It has previously been reported that the $\mathrm{BH}$ mass, $M_{\mathrm{BH}}$, and the Galactic center distance, $D_{\odot}$, show some correlation (Gravity Collaboration 2018b; Do et al. 2019). We here adopted the distance to the Galactic center as a fixed parameter, $D_{\odot}=$ $8 \mathrm{kpc}$. Instead, as we described, we seek a best-fit value for $M_{\mathrm{BH}}$ . It may therefore in principle have some effect on the inferred values if $D_{\odot}$ and $M_{\mathrm{BH}}$ are not considered together as adjustable parameters. However, Table 1 shows that our inferred values for the parameters of the $\mathrm{BH}$ model agree with those reported in previous analyses, including the $\mathrm{BH}$ mass, see, for instance, Gravity Collaboration (2018b) and Do et al. (2019).

Because of the regular initial condition that is applied to solve the equations of the extended RAR model, that is, $\rho(r=$ $0)=$ const. (see Fig. 1 and Appendix A for details), the DM quantum-core is not directly comparable with a $\mathrm{BH}$, which is characterized by a central singularity. However, it is possible to compare the mass that causes the innermost Keplerian behavior (i.e., the power law $\propto r^{-1 / 2}$ in the velocity curve) of orbiting objects in both scenarios. In the RAR model, the Keplerian behavior arises just outside the core radius (see Fig. 1). The corresponding "Keplerian mass", $M_{K}$, which describes the Keplerian trend, is slightly higher than the DM core mass $M_{\mathrm{c}}$ because of the slight mass contribution along the sharp density drop. For larger radii, already in the diluted plateau density, the mass contribution to $M_{K}$ is negligible, until the Keplerian trend ends at about few $1 \times 10^{2}$ pc (curiously, this is at the peak of the bulge velocity curve, see Fig. 1). For a quantum-core mass of $M_{c}=3.5 \times 10^{6} M_{\odot}$, we find the corresponding Keplerian mass
$M_{K}=4.048 \times 10^{6} M_{\odot}$. This value is indeed very similar to the value inferred for the $\mathrm{BH}$ scenario, $M_{\mathrm{BH}}=4.075 \times 10^{6} M_{\odot}$, and should be kept in mind (in addition to $M_{\mathrm{c}}$ ) when the two models are compared regarding the (stellar) dynamics in the surroundings of Sgr A*

We present the equations of motion for the general spherically symmetric metric and the procedure we used to fit the observational data of the apparent orbit and line-of-sight radial velocity (i.e., the redshift function) in both scenarios in Appendix C. Figure 2 shows the results of these two theoretical scenarios and how they compare with the observational data of the orbit (observed right ascension, $X$, and declination, $Y$ ) for the case of S2. The comparison with the data of the line-ofsight radial velocity is shown in Fig. 3. It is already noticeable by visual inspection of the residuals that both theoretical models can explain the observational data for the orbit with similar accuracy. The reduced- $\chi^{2}$ of the model data fit of the $S 2$ radial velocity $\left(\bar{\chi}_{z}^{2}\right)$ and orbit $\left(\bar{\chi}_{X}^{2}\right.$ and $\left.\bar{\chi}_{Y}^{2}\right)$ leads to a comparable mean for both scenarios (with some preference for the RAR model): $\left\langle\bar{\chi}^{2}\right\rangle_{\mathrm{RAR}} \approx 3.072,\left\langle\bar{\chi}^{2}\right\rangle_{\mathrm{BH}} \approx 3.359$. We refer to Table 1 for the model parameters and to Appendix $\mathrm{C}$ for details of the fitting procedure.

The situation becomes even more interesting in the analogous analysis made for G2. As shown in Plewa et al. (2017), Gillessen et al. (2019), the radial velocity of the G2 orbit is slower than the velocity predicted by the geodesic motion in the gravitational field of the massive $\mathrm{BH}$. We therefore propose that G2 is slowed down by a drag force caused by an accretion flow onto the massive $\mathrm{BH}$ over which $\mathrm{G} 2$ should move. The novel major result is that a geodesic in the gravitational field of the DM profile of the extended RAR model naturally predicts this slowing down (see Figs. 4 and 5 and Table 1). The higher G2 deceleration arises because it moves in the gravitational field that is produced by the spatially varying mass profile of the fermionic DM. This deceleration effect is instead negligible in the case of S2 because of the shape of the orbit, more precisely, because of its size. From its pericenter at $\sim 0.6 \mathrm{mpc}$ to apocenter at $\sim 10 \mathrm{mpc}$ (see Table 1), S2 moves only a short distance in which the density of the fermionic DM varies considerably less than in the G2 case. The orbit of G2, from its pericenter at $\sim 0.8 \mathrm{mpc}$ 

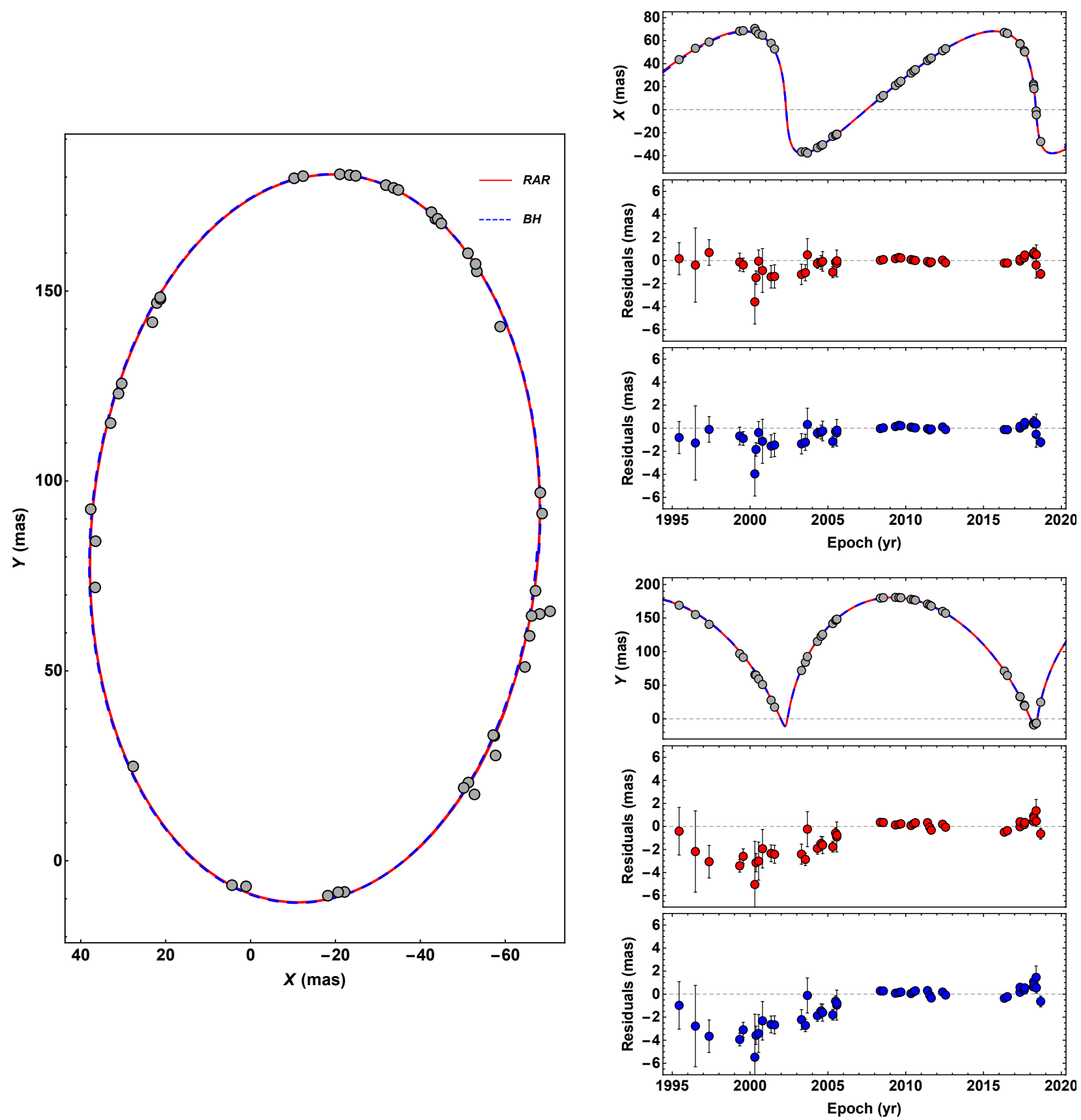

Fig. 2. Theoretical and observed orbit of S2 around Sgr A*. Left panel: orbit ( $X$ vs. $Y$ ), right panel: $X$ and $Y$ position as a function of time and the respective residuals of the best-fit for each model. The theoretical models are calculated by solving the equations of motion of a test particle in the gravitational field of (1) a Schwarzschild BH of $4.075 \times 10^{6} M_{\odot}$ (dashed blue curves), and (2) the DM distribution obtained from the extended RAR model for $56 \mathrm{keV}$ fermions (red curves). The mass of the quantum core in the RAR model is $3.5 \times 10^{6} M_{\odot}$. Table 1 shows the parameters of each model. We used the observational data reported in Do et al. (2019).

to its apocenter at $\sim 85 \mathrm{mpc}$, crosses a much larger region where the DM density drastically drops off from $\sim 1 \times 10^{15} M_{\odot} \mathrm{pc}^{3}$ to $\sim 1 M_{\odot} \mathrm{pc}^{3}$ (see Fig. 1).

\section{S2 gravitational redshift}

The instruments on the ESO Very Large Telescope (VLT) SINFONI, NACO, and more recently, GRAVITY, have accumulated exquisite data on the radial velocity (the redshift function) and motion of S2 for about three decades (Gillessen et al. 2017; Gravity Collaboration 2018b). This has allowed the recent obser- vational detection of the combined gravitational redshift and relativistic transverse Doppler effect for S2 by the GRAVITY Collaboration (Gravity Collaboration 2018b).

The total Doppler shift $z(r)$ is a combination of the gravitational redshift and the relativistic Doppler shift. The GRAVITY Collaboration (Gravity Collaboration 2018b) uses the 2PN expansion of the redshift function for the case of a test particle around a Schwarzschild BH. We now summarize their treatment and refer to Zucker et al. (2006), Do et al. (2019) for its details, and we refer to Appendix B for details on the full general relativistic treatment and a derivation of the $2 \mathrm{PN}$ approximation. At 


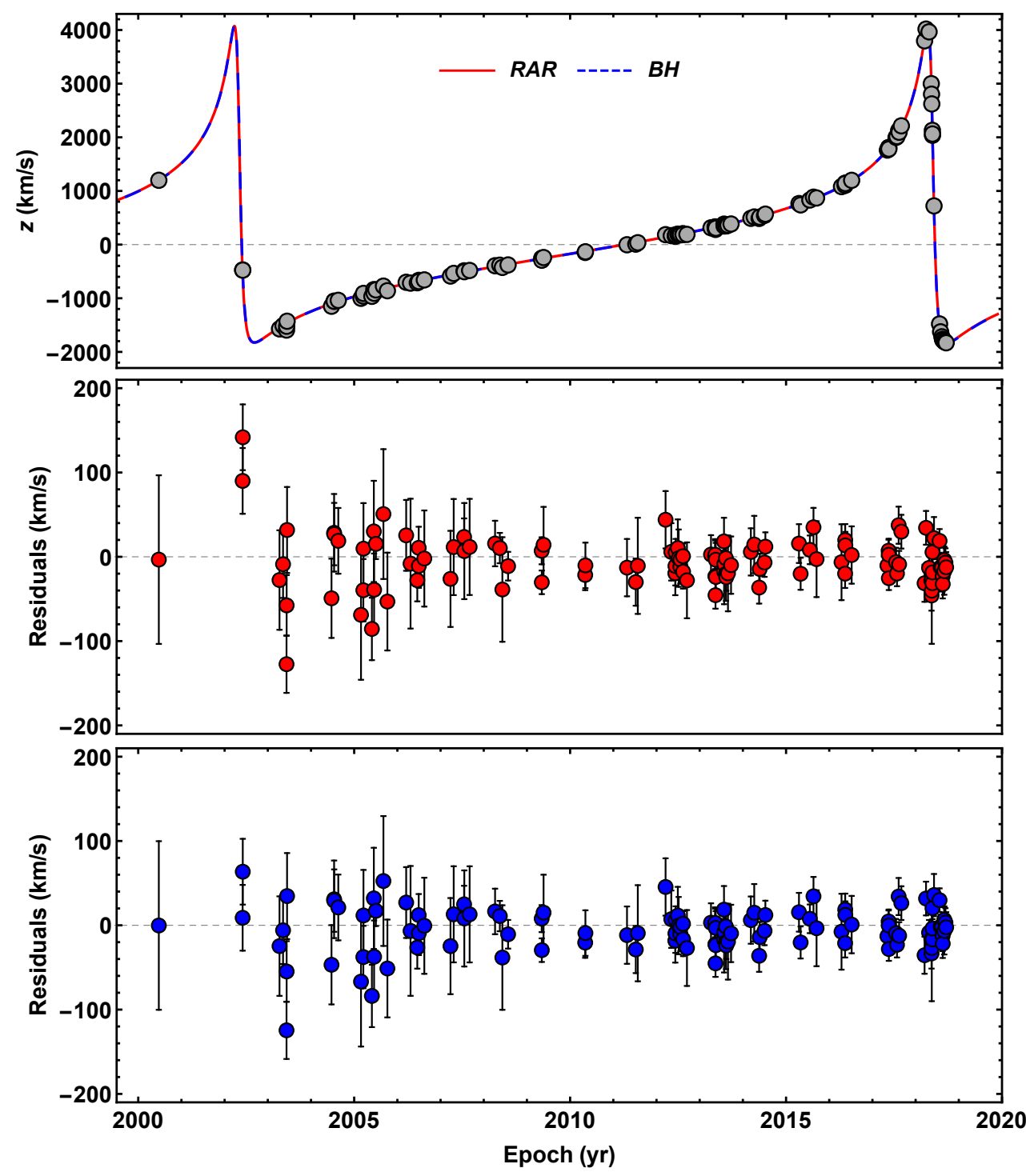

Fig. 3. Theoretical and observed lineof-sight radial velocity (i.e., the redshift function $z$; see Appendix B) of S2. The theoretical models are calculated by solving the equations of motion of a test particle in the gravitational field of (1) a Schwarzschild $\mathrm{BH}$ of $4.075 \times 10^{6} M_{\odot}$ (dashed blue curves), and (2) the DM distribution obtained from the extended RAR model for $56 \mathrm{keV}$ fermions (red curves). The mass of the quantum core in the RAR model is $3.5 \times 10^{6} M_{\odot}$. Table 1 shows the parameters of each model. We used the observational data reported in Do et al. (2019).
2PN order, the redshift function is $z(r) \approx z_{g}(r)+z_{D}(r)+O\left(1 / c^{2}\right)$. The first term $z_{g}$ is the $2 \mathrm{PN}$ expression of the pure gravitational redshift $z_{g}(r)=\sqrt{g_{00}(R) / g_{00}(r)}-1 \approx M_{B H} / r$, where $r$ is the position of the emitted photon (emitter, or source), $R$ is the position of the receiver, and $g_{00}$ is the $0-0$ component of the spacetime metric. Because $R=D_{\odot}=8 \mathrm{kpc}$ is the distance of the Sun to the Galactic center, $r \ll R$, so that we safely approximated $r / R \rightarrow 0$. The second term $z_{D}$ of the $2 \mathrm{PN}$ redshift can be split into the Keplerian (Newtonian) contribution, $z_{K}(r)$, and the purely relativistic transverse Doppler shift, $z_{\mathrm{tD}}$, that is, $z_{D}(r) \approx z_{K}(r)+z_{\mathrm{tD}}(r)$. Here, $z_{K}(r)=\boldsymbol{v} \cdot \boldsymbol{n}$, where $\boldsymbol{n}$ is the unity vector in the direction of the line of sight, and $z_{\mathrm{tD}}(r)=v(r)^{2} / 2$ (see Appendix B). To summarize, at 2PN order, $z(r)=z_{K}(r)+z_{\mathrm{GR}}(r)$, where $z_{\mathrm{GR}}(r)=z_{\mathrm{tD}}(r)+z_{g}(r)$ is the total general relativistic correction. Therefore the deviation from a purely Newtonian behavior can be measured by the general relativistic excess of the radial velocity, $\Delta z(r) \equiv z(r)-z_{K}(r)=z_{\mathrm{GR}}(r)$ (Gravity Collaboration 2018b). Because the extended-RAR model is fully general relativistic, we used the full general relativistic expression of the redshift function and the corresponding general relativistic excess (see Appendix B for details).

Figures 3 and 5 show the redshift function $z$ computed in full general relativity for the massive $\mathrm{BH}$ and the extended RAR model for S2 and G2, respectively. In the top panel of Fig. 6, we show the redshift function $z$ together with the corresponding Keplerian contribution $z_{K}$ for S2 in the two models. The bottom panels show the corresponding general relativistic excess, $\Delta z$. All these plots show that both models fit the data with comparable accuracy. The reduced $\chi^{2}$ for the redshift function for this set of parameters is $\bar{\chi}_{z, \mathrm{RAR}}^{2} \approx 1.28$ and $\bar{\chi}_{z, \mathrm{BH}}^{2} \approx 1.04$; see Appendix $\mathrm{C}$ for details of the calculation of $\bar{\chi}^{2}$. It is important to mention that both models contain sets of parameters with slightly different values than those that we presented in Table 1, which produce $\bar{\chi}_{z, \mathrm{RAR}}^{2} \approx \bar{\chi}_{z, \mathrm{BH}}^{2} \approx 1$. However, these models slightly increase the $\bar{\chi}_{X}^{2}$ and $\bar{\chi}_{Y}^{2}$ and therefore increase the mean $\left\langle\bar{\chi}^{2}\right\rangle$.

\section{Discussion and conclusions}

The vast amount of high-precision data (position and velocity) collected in the past decade of objects orbiting Sgr A*, such as S2 and G2, offers an unprecedented opportunity to test alternative scenarios to the central $\mathrm{BH}$ in our Galaxy. In the work here, this motivation is twofold. First, it has recently been shown (Argüelles et al. 2019b,a, 2018) that fermionic DM, which self-consistently accounts for the Pauli principle and 

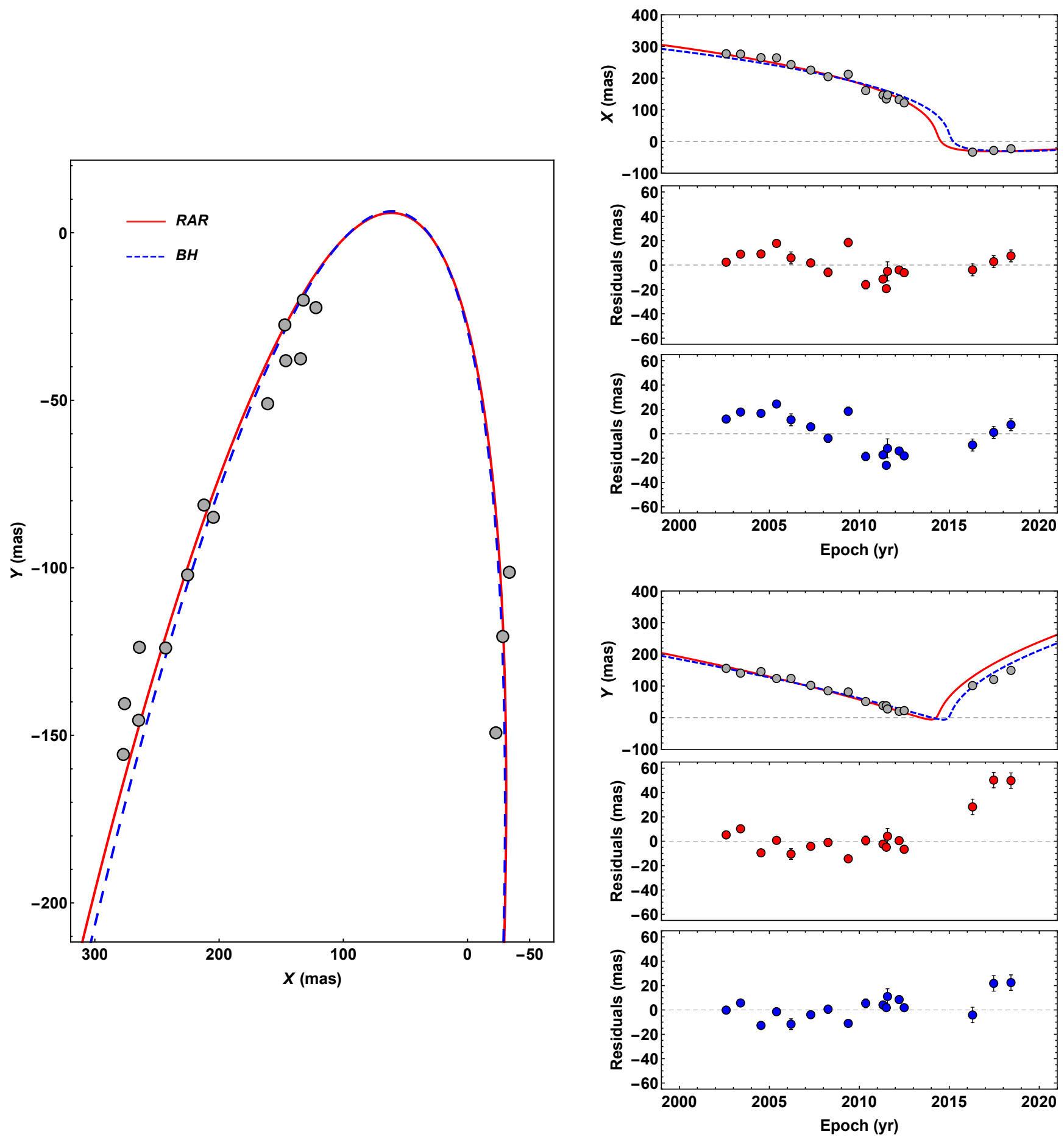

Fig. 4. Theoretical and observed orbit of G2 around Sgr A*. Left panel: orbit ( $X$ vs. $Y$ ), right panel: $X$ and $Y$ position as a function of time and the respective residuals of the best-fit for each model. The theoretical models are calculated by solving the equations of motion of a test particle in the gravitational field of (1) a Schwarzschild BH of $4.075 \times 10^{6} M_{\odot}$ (dashed blue curves), and (2) the DM distribution obtained from the extended RAR model for $56 \mathrm{keV}$ fermions (red curves). The mass of the quantum core in the RAR model is $3.5 \times 10^{6} M_{\odot}$. Table 1 shows the parameters of each model. The observational data are taken from Phifer et al. (2013), Plewa et al. (2017), and Gillessen et al. (2019).

particle escape effects in the underlying phase-space DF at DM halo formation, leads to novel dense core - diluted halo profiles where the degenerate core can produce analogous gravitational effects of a central BH. Second, the post-pericenter passage of $\mathrm{G} 2$ challenges the $\mathrm{BH}$ scenario because in order to explain the G2 data within this picture, Gillessen et al. (2019) had to introduce an ad hoc drag force acting onto $\mathrm{G} 2$, caused by its motion through an accretion flow. In addition, for this drag-force hypothesis to work, it is necessary that G2 be a gas cloud. This scenario contrasts with the observations and results of Witzel et al. (2014), who ruled out the gas cloud composition in favor of a stellar nature. Moreover, even if G2 is assumed to be a gas cloud, and if a radiatively inefficient accretion flow (RIAF) is also assumed (as done in Gillessen et al. 2019), the 

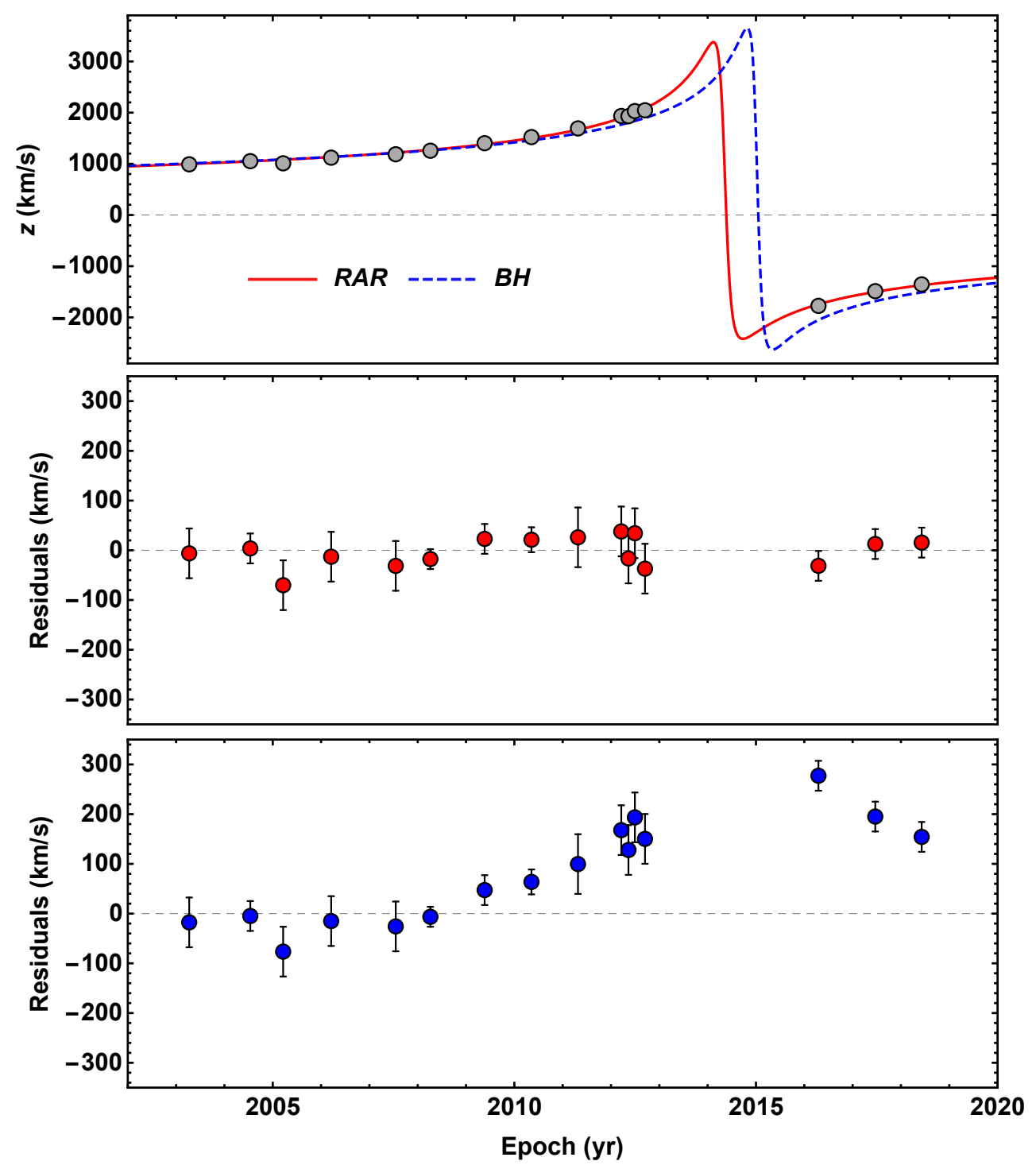

Fig. 5. Theoretical and observed lineof-sight radial velocity (i.e., the redshift function $z$; see Appendix B) of G2. The theoretical models are calculated by solving the equations of motion of a test particle in the gravitational field of (1) a Schwarzschild BH of $4.075 \times 10^{6} M_{\odot}$ (dashed blue curves), and (2) the DM distribution obtained from the extended RAR model for $56 \mathrm{keV}$ fermions (red curves). The mass of the quantum core in the RAR model is $3.5 \times 10^{6} M_{\odot}$. Table 1 shows the parameters of each model. The observational data are taken from Phifer et al. (2013), Plewa et al. (2017), and Gillessen et al. (2019). strength of the drag force onto $\mathrm{G} 2$ that is required to explain the post-pericenter observations implies an ambient density $n_{0} \sim$ few $10^{3} \mathrm{~cm}^{-3}$ at $\sim 10^{3} r_{\text {Sch. }}$. However, a density value like this at these pericenter scales exceeds the upper bound found in recent high-resolution numerical simulations by nearly one order of magnitude ${ }^{1}$ (Steinberg et al. 2018). An upper bound like this has been obtained from the constraint that G2 is not tidally disrupted at its pericenter passage.

For the core-halo DM profiles, formation scenarios in which the quantum nature of the particle is considered (i.e., either bosonic or fermionic) are still an open field of research, and our aim here is to provide a further (precision) test for fermionic models. Joint observational tests based on additional physics, for example, strong lensing (Gómez et al. 2016) or DM-active neutrino interactions (Penacchioni et al. 2020), can help in unambiguously probing the existence of a central fermionic DM concentration in the allowed region of the extended RAR model parameter space. The results shown here imply that this free parameter space is slightly reduced with respect to the space described in Argüelles et al. (2018). For fermion masses

\footnotetext{
1 There are systematic uncertainties in the estimation of $n_{0}$ in Gillessen et al. (2019) mainly due to the unknown size of the putative gas cloud, the density profile, and the physics of the accretion process.
}

below $56 \mathrm{keV}$, the size of the $\mathrm{DM}$ core increases, and there is also orbital precession. Data of the orbital precession of S2 (Gravity Collaboration 2020) might therefore further constrain the allowed range of the fermion mass. The other free parameters are well within the allowed range as broadly constrained in Arguielles et al. (2019a) for each galaxy type.

We have used the existing observational data of S2, including the total Doppler shift, which has both special and general relativistic contributions, and the orbit in the plane of sky and its radial velocity. We solved the equations of motion for a test particle (S2 and G2) in the gravitational field produced by two cases of interest: (1) the central massive $\mathrm{BH}$ hypothesis, for which we used the Schwarzschild metric, and (2) the fermionic DM hypothesis within the extended-RAR model, which leads to a DM core-halo profile that in turn leads to a metric obtained from the extended RAR model equilibrium equations following the treatment in Argüelles et al. (2018) and summarized in Appendix A. We refer to Appendix $\mathrm{C}$ for details of the equations of motion and the procedure to obtain the model parameters from the fitting of the observational data. For S2, the massive $\mathrm{BH}$ model and the RAR model can both explain all the observational data (orbit and velocity) with comparable accuracy, but the RAR model is preferable with a lower $\left\langle\bar{\chi}^{2}\right\rangle$; see Table 1, Figs. 2, and 3, including the general relativistic redshift, see Fig. 6 . In the case 


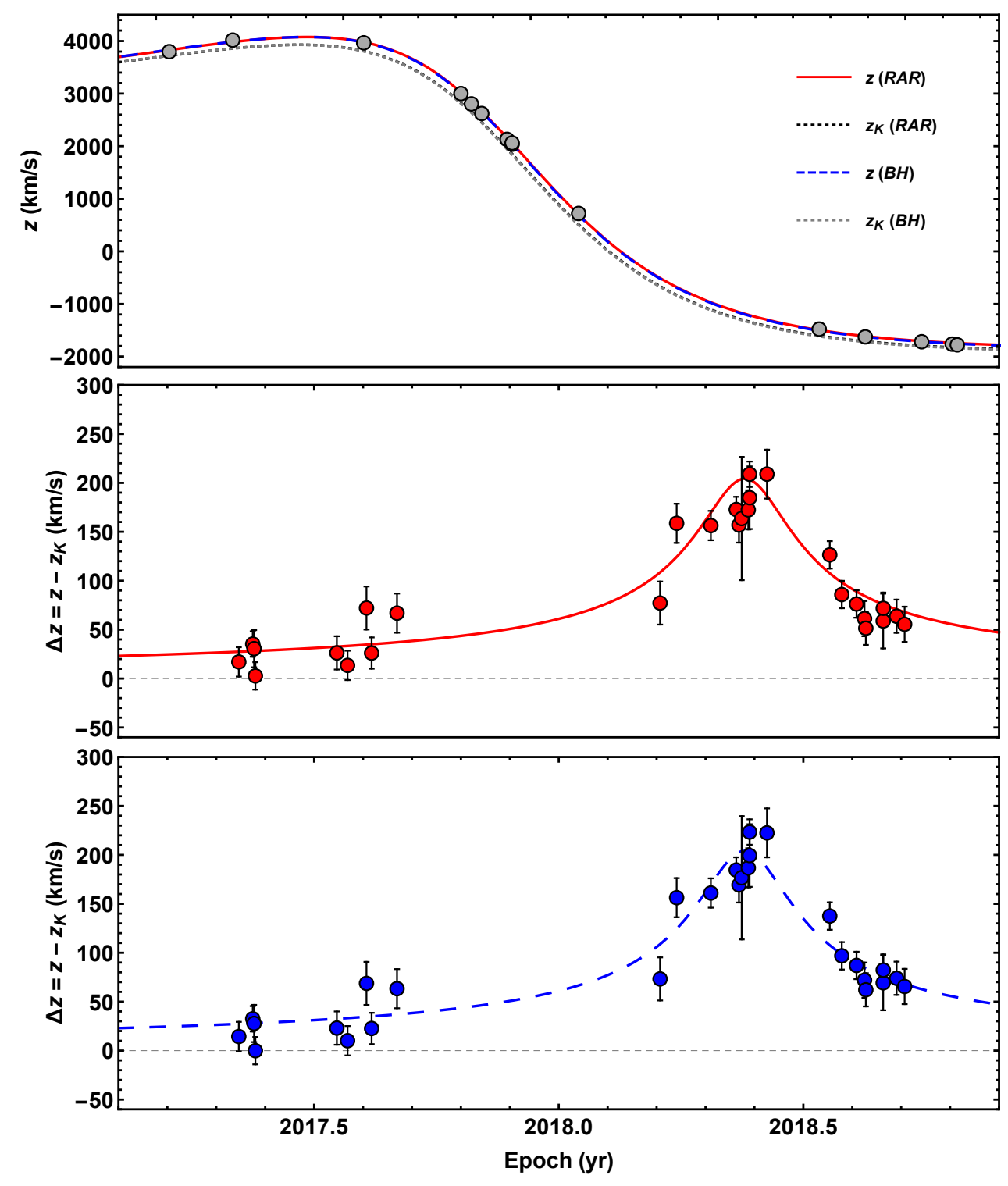

Fig. 6. Redshift function $z$ (top panel) and redshift function "excess" (middle panel for the RAR model and lower panel for the central massive $\mathrm{BH}$ model) with respect to the Keplerian (Newtonian) contribution, i.e., $\Delta z=z-z_{K}$ (see Appendix B), for the S2 motion at about its pericenter passage. The theoretical models are calculated by solving the equations of motion of a test particle in the gravitational field of (1) a Schwarzschild BH of $4.075 \times 10^{6} M_{\odot}$ (dashed blue curves), and (2) the DM distribution obtained from the extended RAR model for $56 \mathrm{keV}$ fermions (red curves). The mass of the quantum core in the RAR model is $3.5 \times 10^{6} M_{\odot}$. Table 1 shows the parameters of each model. of G2, only the RAR model can explain both the orbit and velocity, see Table 1, Fig. 4, and 5.

This remarkable result of the extended-RAR fermion-DM model is further complemented with the successful applicability of its ensuing dense core - diluted halo profile to other galaxy types, from dwarfs to ellipticals (Argüelles et al. 2019a). Moreover, it can be directly linked with the DM-halo formation processes because the RAR model quantum-statistical phase-space distribution (see Eq. (A.1)) is not given ad hoc, but can be obtained as a (quasi-) stationary solution of a generalized thermodynamic Fokker-Planck equation for fermions (Chavanis 2004). This includes the physics of collisionless (violent) relaxation and evaporation, which is appropriate for nonlinear structure formation. These phase-space distributions have been shown to fulfill a maximization (coarse-grained) entropy principle (second law of thermodynamics) during the (collisionless) relaxation process until the halo reaches the currently observed steady state.

Our results provide strong observational support to the quantum-core hypothesis as an alternative to the massive $\mathrm{BH}$ hypothesis in Sgr A* (Argüelles et al. 2019b, 2018), and also to the fermionic nature of DM. In this line, it is desirable to further test the presence of fermionic DM concentrations in our galactic core from existing luminosity constraints on the variability of the compact radio source $\mathrm{Sgr} \mathrm{A}^{*}$, in addition to the dynamical constraints. A study like this goes beyond the scope of our work, which is devoted to the orbital dynamics of some of the closest objects to Sgr A* and with accurate astrometric data. We would like to recall, however, that the gravitational potentials produced by a $\mathrm{BH}$ and by a mmost compact (stable) DM quantum core for a fermion mass of about $100 \mathrm{keV}$ practically coincide at distances $r \gtrsim 10 r_{\mathrm{Sch}}$ (see Gómez et al. 2016, for details). The dynamics of baryonic matter and its emission associated with its motion at these scales is thus not expected to differ much between the two pictures. Differences might occur in the innermost regions owing to the transparency of the DM core, which might lead to differences in the lensing properties (Gómez et al. 2016) and possibly to any accretion process at these small scales. Moreover, although the emission around $\mathrm{Sgr} \mathrm{A} *$ is often univocally associated with a particular accretion flow (extremely underluminous when compared to typical accretion expectations), this is not confirmed by the observational data, and indeed, alternative mechanisms or explanations for the observed radiation exist (see, e.g., Yuan \& Narayan 2014 , for a review on this subject). As of today, the most reliable 
observational data that allow us to prove and test the validity of alternative models for Sgr A*, such as we presented here, are the precision measurements of the orbital dynamics, together with the validity and demonstrated precision of general relativity. We look forward to the public release of the latest data by the GRAVITY Collaboration on S2 and G2 (e.g., Gillessen et al. 2019), which will serve to further test our theoretical prediction (e.g., Fig. 5). We have shown the results for a fermion mass of $56 \mathrm{keV}$, a value that is safely higher than the lower limit of $48 \mathrm{keV}$ estimated in Argüelles et al. (2018), by equating the DM core radius to the upto-then reported pericenter distance of S2. The lower the fermion mass, the larger the size of the DM core, and vice versa. It is therefore worthwhile to explore whether the data of S2 and G2 together might further constrain the allowed range of fermion masses. This investigation, however, goes beyond the scope of this work, and might be a topic of joint collaboration.

The DM fermion mass of $56 \mathrm{keV}$ inferred in this work would produce (down to megaparsec scales) the same standard $\Lambda$ CDM power spectrum, hence providing the expected large-scale structure (Boyarsky et al. 2009a). Because the fermion mass is higher than $>5 \mathrm{keV}$, it does not contradict constraints from the Lyman$\alpha$ forest (Boyarsky et al. 2009b; Viel et al. 2013; Irsic et al. 2017) and the number of Milky Way satellites (Tollerud et al. 2008). Furthermore, for the present fermion mass $m c^{2}=56 \mathrm{keV}$, the critical mass for gravitational collapse of the DM quantum core $\left(M_{\mathrm{c}}^{\mathrm{cr}} \sim m_{\mathrm{Pl}}^{3} / m^{2}\right.$, with $m_{\mathrm{Pl}}$ the Planck mass) into a $\mathrm{BH}$ is about $10^{8} M_{\odot}$, providing a viable formation scenario for the observed central supermassive $\mathrm{BH}$ in active galaxies such as M87. A supermassive $\mathrm{BH}$ of $\sim 10^{9} \mathrm{M}_{\odot}$ can form starting from a $\sim 10^{8} M_{\odot} \mathrm{BH}$ seed and accrete $\lesssim 1 \%$ of the (baryonic and/or DM) galactic environment of $\sim 10^{12} M_{\odot}$. Over cosmological timescales, this would be achieved without unrealistic superEddington accretion rates, while providing a new framework for studying the poorly understood formation and growth scenarios of supermassive BH seeds in the cosmological high-redshift Universe.

Acknowledgements. We thank the Referee and the Editor for their helpful suggestions which have improved the presentation of our results. E.A.B-V. thanks financial and research support from COLCIENCIAS under the program Becas Doctorados Nacionales 727, the International Center for Relativistic Astrophysics Network (ICRANet), Universidad Industrial de Santander (UIS) and the International Relativistic Astrophysics Ph.D Program (IRAP-PhD). C.R.A has been supported by CONICET and Secretary of Science and Technology of FCAG.

\section{References}

Argüelles, C. R., Mavromatos, N. E., Rueda, J. A., \& Ruffini, R. 2016, J. Cosmol. Astropart. Phys., 4, 038

Argüelles, C. R., Krut, A., Rueda, J. A., \& Ruffini, R. 2018, Phys. Dark Universe, 21,82

Argüelles, C. R., Krut, A., Rueda, J. A., \& Ruffini, R. 2019a, Phys. Dark Universe, 24, 100278

Argüelles, C. R., Krut, A., Rueda, J. A., \& Ruffini, R. 2019b, Int. J. Mod. Phys. D, 28, 1943003

Bilic, N., Munyaneza, F., Tupper, G. B., \& Viollier, R. D. 2002, Prog. Part. Nucl. Phys., 48, 291

Boyarsky, A., Ruchayskiy, O., \& Shaposhnikov, M. 2009a, Annu. Rev. Nucl. Part. S., 59, 191

Boyarsky, A., Lesgourgues, J., Ruchayskiy, O., \& Viel, M. 2009b, Phys. Rev. Lett., 102, 201304

Chavanis, P.-H. 2004, Phys. A, 332, 89

Chavanis, P.-H., Lemou, M., \& Méhats, F. 2015, Phys. Rev. D, 92, 123527

Chu, D. S., Do, T., Hees, A., et al. 2018, ApJ, 854, 12

de Vega, H. J., Salucci, P., \& Sanchez, N. G. 2014, MNRAS, 442, 2717

Do, T., Hees, A., Ghez, A., et al. 2019, Science, 365, 664

Fehlberg, E. 1970, Computing, 6, 61

Genzel, R., Eisenhauer, F., \& Gillessen, S. 2010, Rev. Mod. Phys., 82, 3121

Ghez, A. M., Salim, S., Weinberg, N. N., et al. 2008, ApJ, 689, 1044

Gibbons, S. L. J., Belokurov, V., \& Evans, N. W. 2014, MNRAS, 445, 3788

Gillessen, S., Eisenhauer, F., Fritz, T. K., et al. 2009, ApJ, 707, L114

Gillessen, S., Plewa, P. M., Eisenhauer, F., et al. 2017, ApJ, 837, 30

Gillessen, S., Plewa, P. M., Widmann, F., et al. 2019, ApJ, 871, 126

Gómez, L. G., Argüelles, C. R., Perlick, V., Rueda, J. A., \& Ruffini, R. 2016, Phys. Rev. D, 94, 123004

Gravity Collaboration (Abuter, R., et al.) 2018a, A\&A, 618, L10

Gravity Collaboration (Abuter, R., et al.) 2018b, A\&A, 615, L15

Gravity Collaboration (Abuter, R., et al.) 2020, A\&A, 636, L5

Irsic, V., Viel, M., Haehnelt, M. G., et al. 2017, Phys. Rev. D, 96, 023522

Klein, O. 1949, Rev. Mod. Phys., 21, 531

Mavromatos, N. E., Argüelles, C. R., Ruffini, R., \& Rueda, J. A. 2017, Int. J. Mod. Phys. D, 26, 1730007

Merafina, M., \& Ruffini, R. 1989, A\&A, 221, 4

Penacchioni, A. V., Civitarese, O., \& Argüelles, C. R. 2020, Eur. Phys. J. C, 80, 183

Phifer, K., Do, T., Meyer, L., et al. 2013, ApJ, 773, L13

Plewa, P. M., Gillessen, S., Pfuhl, O., et al. 2017, ApJ, 840, 50

Randall, L., Scholtz, J., \& Unwin, J. 2017, MNRAS, 467, 1515

Ruffini, R., Argüelles, C. R., \& Rueda, J. A. 2015, MNRAS, 451, 622

Siutsou, I., Argüelles, C. R., \& Ruffini, R. 2015, Astron. Rep., 59, 656

Sofue, Y. 2013, PASJ, 65, 118

Steinberg, E., Sari, R., Gnat, O., et al. 2018, MNRAS, 473, 1841

Tollerud, E. J., Bullock, J. S., Strigari, L. E., \& Willman, B. 2008, ApJ, 688, 277

Tolman, R. C. 1930, Phys. Rev., 35, 904

Viel, M., Becker, G. D., Bolton, J. S., \& Haehnelt, M. G. 2013, Phys. Rev. D, 88, 043502

Witzel, G., Ghez, A. M., Morris, M. R., et al. 2014, ApJ, 796, L8

Yuan, F., \& Narayan, R. 2014, ARA\&A, 52, 529

Zucker, S., Alexander, T., Gillessen, S., Eisenhauer, F., \& Genzel, R. 2006, ApJ, 639, L21 


\section{Appendix A: Extended Ruffini-Argüelles-Rueda model}

The extended RAR model conceives the DM in galaxies as a general relativistic self-gravitating system of massive fermions (spin 1/2) in hydrostatic and thermodynamic equilibrium. It uses an equation of state (EOS) that takes into account (i) relativistic effects of the fermionic constituents, (ii) finite temperature effects, and (iii) particle escape effects at large momentum $(p)$ through a cutoff in the Fermi-Dirac distribution $f_{\mathrm{c}}$,

$f_{\mathrm{c}}\left(\epsilon \leq \epsilon_{\mathrm{c}}\right)=\frac{1-e^{\left(\epsilon-\epsilon_{\mathrm{c}}\right) / k T}}{e^{(\epsilon-\mu) / k T}+1}, \quad f_{\mathrm{c}}\left(\epsilon>\epsilon_{\mathrm{c}}\right)=0$,

which differs from the original RAR model version (see Sect. 2) only in condition (iii). Where $\epsilon=\sqrt{c^{2} p^{2}+m^{2} c^{4}}-m c^{2}$ is the particle kinetic energy, $\mu$ is the chemical potential from which the particle rest-energy is subtracted, $T$ is the temperature, $k$ is the Boltzmann constant, $c$ is the speed of light, and $m$ is the fermion mass. The stress-energy tensor is that of a perfect fluid whose density and pressure are associated with this distribution function,

$$
\begin{aligned}
& \rho=m \frac{2}{h^{3}} \int_{0}^{\epsilon_{\mathrm{c}}} f_{\mathrm{c}}(p)\left(1+\frac{\epsilon(p)}{m c^{2}}\right) \mathrm{d}^{3} p, \\
& P=\frac{1}{3} \frac{4}{h^{3}} \int_{0}^{\epsilon_{\mathrm{c}}} f_{\mathrm{c}}(p) \epsilon \frac{1+\epsilon(p) / 2 m c^{2}}{1+\epsilon(p) / m c^{2}} \mathrm{~d}^{3} p .
\end{aligned}
$$

For the spherically symmetric spacetime metric,

$\mathrm{d} s^{2}=g_{00}(r) \mathrm{d} t^{2}-g_{11}(r) \mathrm{d} r^{2}-r^{2}\left(\mathrm{~d} \theta^{2}+\sin ^{2} \theta \mathrm{d} \phi^{2}\right)$,

where $(r, \theta, \phi)$ are the spherical coordinates. Using $g_{00}(r)=e^{\nu(r)}$, the Tolman (Tolman 1930), Klein (Klein 1949), and the cutoff (Merafina \& Ruffini 1989) conditions of thermodynamic equilibrium and energy conservation are

$e^{v / 2} T=$ constant,

$e^{v / 2}\left(\mu+m c^{2}\right)=$ constant,

$e^{v / 2}\left(\epsilon+m c^{2}\right)=$ constant.

The Einstein equations together with the conditions given by Eqs. (A.5)-(A.7) form a coupled system of integro-differential equations,

$$
\begin{aligned}
& \frac{\mathrm{d} \hat{M}}{\mathrm{~d} \hat{r}}=4 \pi \hat{r}^{2} \hat{\rho}, \\
& \frac{\mathrm{d} \theta}{\mathrm{d} \hat{r}}=-\frac{1-\beta_{0}\left(\theta-\theta_{0}\right)}{\beta_{0}} \frac{\hat{M}+4 \pi \hat{P} \hat{r}^{3}}{\hat{r}^{2}(1-2 \hat{M} / \hat{r})}, \\
& \frac{\mathrm{d} v}{\mathrm{~d} \hat{r}}=\frac{2\left(\hat{M}+4 \pi \hat{P} \hat{r}^{3}\right)}{\hat{r}^{2}(1-2 \hat{M} / \hat{r})}, \\
& \beta(\hat{r})=\beta_{0} e^{\frac{\nu_{0}-v(\hat{r})}{2}}, \\
& W(\hat{r})=W_{0}+\theta(\hat{r})-\theta_{0},
\end{aligned}
$$

where the subscript " 0 " stands for variable evaluated at $r=0$, and we have introduced dimensionless quantities: $\beta=k T /\left(m c^{2}\right)$, $\theta=\mu /(k T), W=\epsilon_{\mathrm{c}} /(k T), \hat{r}=r / \chi, \hat{M}=G M /\left(c^{2} \chi\right), \hat{\rho}=$ $G \chi^{2} \rho / c^{2}, \hat{P}=G \chi^{2} P / c^{4}$, where $\chi=2 \pi^{3 / 2}(\hbar / m c)\left(m_{\mathrm{Pl}} / m\right)$, being $m_{\mathrm{Pl}}=\sqrt{\hbar c / G}$ the Planck mass.

This system is solved for appropriate boundary conditions, $\left[M(0)=0, \theta(0)=\theta_{0}, \beta(0)=\beta_{0}, v(0)=0, W(0)=W_{0}\right]$, for different DM particle masses $m$ to find a solution consistent with the DM halo observables of a given galaxy. The RAR model equations are solved for positive central degeneracy parameters (i.e., $\theta_{0}>10$ ) in order to ensure that the Pauli principle is fulfilled within the central core, as demonstrated in Ruffini et al. (2015) and Arguielles et al. (2018). This property implies as a consequence RAR DM profiles that develop a dense core diluted halo morphology, where the central core is governed by Fermi-degeneracy pressure, while the outer halo holds against gravity by thermal pressure (resembling the Burkert or King profiles, as shown in Argüelles et al. 2018, 2019a). The extended RAR model is the more general of its kind because it does not work under the full Fermi-degeneracy approximation as in Randall et al. (2017), nor in the diluted-Fermi regime (de Vega et al. 2014).

The case of the Milky Way has recently been analyzed in Argüelles et al. (2018). We adopted a similar boundary condition problem as solved in Argüelles et al. (2018), with the only difference that we now allowed the dense DM core $M_{\mathrm{c}}$ to vary until the mean reduced $-\chi^{2}$ of the $S 2$ data fit (see Appendix C) achieved the minimum. That is, we considered (i) a DM halo mass with observationally inferred values at two different radial locations in the Galaxy: a DM halo mass $M(r=40 \mathrm{kpc})=2 \times 10^{11} M_{\odot}$ (Gibbons et al. 2014) and $M(r=12 \mathrm{kpc})=5 \times 10^{10} M_{\odot}$ (Sofue 2013); and (ii) a DM dense quantum core to have a mass $M\left(r=r_{\mathrm{c}}\right) \equiv M_{\mathrm{c}}=3.5 \times 10^{6} M_{\odot}$ with $r_{\mathrm{c}}$ smaller than the pericenter of star S2, resulting in $r_{\mathrm{c}} \approx 0.4 \mathrm{mpc}$ by the extended RAR model free parameters given in Fig. 1. While the halo condition (i) exactly follows the method that was used in Argüelles et al. (2018), the latter condition (ii) explicitly request the quantum DM core to substitute the massive BH scenario while minimizing the mean reduced $-\chi^{2}$ for the $S 2$ data fit (see Appendix C). We thus have three boundary conditions for three free RARmodel parameters $\left(\beta_{0}, \theta_{0}\right.$, and $\left.W_{0}\right)$ for a given particle mass of $m c^{2}=56 \mathrm{keV}$. It is of interest to explore whether the data of $\mathrm{S} 2$ and G2 together can further constrain the allowed range of fermion masses. This investigation, however, goes beyond the scope of the present work. The application of the extended RAR model to other galaxy types from dwarfs and ellipticals to galaxy clusters can be found in Argüelles et al. (2019a).

\section{Appendix B: Total orbital Doppler shift}

The redshift is defined by the ratio between the measured wavelength of a spectral line at emission and reception:

$1+z \equiv \frac{\mathcal{E}_{(\mathrm{em})}}{\mathcal{E}_{(\mathrm{obs})}}=\frac{\lambda_{(\mathrm{obs})}}{\lambda_{(\mathrm{em})}}$

We denote the four-momentum of photons measured by an observer comoving with the emitter, $k_{(\mathrm{em})}^{\mu}$, and the one measured by an observer comoving with the receiver, $k_{(\mathrm{obs})}^{\mu}$. The observer comoving with the emitter has four-velocity $u_{(\mathrm{em})}^{\mu}$, so they measure a photon energy $\mathcal{E}_{(\mathrm{em})}=k_{\mu}^{(\mathrm{em})} u_{(\mathrm{em})}^{\mu}$. Analogously, the observer comoving with the receiver measures a photon energy $\mathcal{E}_{(\mathrm{obs})}=k_{\mu}^{(\mathrm{obs})} u_{\text {(obs) }}^{\mu}$. Therefore, theoretically, we can write Eq. (B.1) as

$1+z=\frac{k_{\mu}^{(\mathrm{em})} u_{(\mathrm{em})}^{\mu}}{k_{\mu}^{(\mathrm{obs})} u_{(\mathrm{obs})}^{\mu}}=\frac{k_{0}^{(\mathrm{em})}}{k_{0}^{(\mathrm{obs})}} \frac{u_{(\mathrm{em})}^{0}+u_{(\mathrm{em})}^{i} n_{i}^{(\mathrm{em})}}{u_{(\mathrm{obs})}^{0}+u_{(\mathrm{obs})}^{i} n_{i}^{(\mathrm{obs})}}$,

where $n^{i}=k^{i} / k^{0}$ are the normalized spatial components of the photon four-momentum. Defining the components of the threevelocity, $v^{i} \equiv u^{i} / u^{0}$, and the Lorentz factor (where the right-hand 
side of the equation below is obtained from the normalization condition $u^{\mu} u_{\mu}=1$ ),

$\gamma=u^{0}=\frac{\mathrm{d} t}{\mathrm{~d} \tau}=\frac{1}{\sqrt{g_{00}-v^{2}}}, \quad v^{2}=-v^{i} v_{i}=-g_{11}\left(v_{r}\right)^{2}+\left(r v_{\phi}\right)^{2}$,

Eq. (B.2) becomes

$1+z=\frac{\gamma_{(\mathrm{em})}}{\gamma_{(\mathrm{obs})}} \frac{1+v_{(\mathrm{em})}^{i} n_{i}^{(\mathrm{em})}}{1+v_{(\mathrm{obs})}^{i} n_{i}^{(\mathrm{obs})}}$,

where we used the fact that along the photon geodesic, $k_{0}$ is conserved.

For the present purpose, we can neglect the motion of the observer reference frame with respect to the one of the center of the gravitational field with sufficient accuracy, that is, $v_{\text {(obs) }}^{i}=0$, and the gravitational field at the observation point, $g_{00}^{\text {(obs) }}=1$ (see, e.g., Do et al. 2019), then $\gamma_{(\mathrm{obs})}=1$, and Eq. (B.4) becomes

$1+z=\gamma(1+\boldsymbol{v} \cdot \boldsymbol{n})$,

where $\boldsymbol{v} \cdot \boldsymbol{n}=v^{i} n_{i}$ is the three-dimensional velocity of the emitter projected onto the direction of the line of sight; this is often called the observed "radial velocity" in the experimental literature, and we relaxed the notation of emitter and receiver because only the emitter is considered to be in motion.

It is important to clarify that the redshift function $z$ is often referred to in the literature as "radial velocity", the velocity in the direction of the line of sight. The latter is actually $\boldsymbol{v} \cdot \boldsymbol{n}$, therefore, as can be seen from Eq. (B.5), the relation between it and $z$ is in general nonlinear.

In general, it is not possible to separate the contributions to $z$ of the gravitational field and of the relative motion of the emitter or receiver, that is, they are combined or mixed in Eq. (B.5). However, this equation already explicitly shows that in the nonrelativistic limit $(\gamma \rightarrow 1)$, the redshift is given only by the so-called Keplerian (Newtonian) contribution, $z \rightarrow z_{K}$, where

$z_{K} \equiv \boldsymbol{v} \cdot \boldsymbol{n}$

The gravitational and relative motion contributions become clearly visible when a post-Newtonian expansion of the redshift is performed. For instance, when the gravitational field is produced by a Schwarzschild $\mathrm{BH}$ of mass $M_{\mathrm{BH}}$, that is, $g_{00}=$ $-1 / g_{11}=1-2 M_{\mathrm{BH}} / r$, the Lorentz factor, up to order $1 / c^{2}$ (i.e., $2 \mathrm{PN}$ order), is

$$
\begin{aligned}
\gamma & \approx\left(1+\frac{M_{\mathrm{BH}}}{r}\right)\left(1+\frac{v^{2} / 2}{1-2 M_{\mathrm{BH}} / r}\right) \\
& \approx\left(1+\frac{M_{\mathrm{BH}}}{r}\right)\left[1+\frac{1}{2} v^{2}\left(1+\frac{2 M_{\mathrm{BH}}}{r}\right)\right] \\
& \approx\left(1+\frac{M_{\mathrm{BH}}}{r}\right)\left(1+\frac{1}{2} v^{2}\right) \approx 1+\frac{1}{2} v^{2}+\frac{M_{\mathrm{BH}}}{r}+O\left(1 / c^{2}\right),
\end{aligned}
$$

which, replaced into Eq. (B.5), leads to the 2PN redshift function:

$z \approx z_{K}+\frac{1}{2} v^{2}+\frac{M_{\mathrm{BH}}}{r}+O\left(1 / c^{2}\right)$.

Equation (B.8) is the expression presented in Zucker et al. (2006) (see Eq. (1) therein), and it is the radial velocity Eq. (S24) in
Do et al. (2019), setting $v_{z 0}=0$ there, and consistent with our assumption of neglecting the relative motion of the gravitational center of mass with respect to the center of the observer's reference frame. The approximate Eq. (B.8) has been used in those works for the analysis of the gravitational contribution to the redshift function in the case of the S2 star.

The GRAVITY Collaboration (Gravity Collaboration 2018b) has claimed the detection of the gravitational redshift in the orbit of the star S2. In practice, they verified the consistency of the data of the redshift function of S2 with the presence of what they call the "general relativistic excess of the radial velocity" (Gravity Collaboration 2018b),

$\Delta z \equiv z-z_{K}$

Equation (B.9) tells that the theoretical excess predicted by general relativity at $2 \mathrm{PN}$ order is

$\Delta z \approx \frac{1}{2} v^{2}+\frac{M_{\mathrm{BH}}}{r}$

which has been shown to be consistent with the data of star S2 (Gravity Collaboration 2018b).

The present RAR model is a fully general relativistic treatment, therefore we used the full redshift function (B.5) in the fit of the observational data (see Appendix C). In this case, the general relativistic excess in the redshift, as defined by Eq. (B.9), reads

$\Delta z=(\gamma-1)\left(1+z_{K}\right)$.

It is manifest in the fully general expression Eq. (B.11) that in the nonrelativistic (Newtonian) limit, $\gamma \rightarrow 1$, the excess vanishes, that is, $\Delta z \rightarrow 0$. It is also easy to verify that Eq. (B.11) reduces to Eq. (B.10) at 2PN order, with the aid of Eq. (B.7).

\section{Appendix C: Equations of motion and orbital parameters of the real and apparent orbits}

\section{C.1. Orbital dynamics}

The equations of motion of the test particle (S2 or G2) in the spherically symmetric metric given by Eq. (A.4), assuming without loss of generality $\theta=\pi / 2$, are

$\dot{t}=\frac{E}{g_{00}(r)}$,

$\ddot{r}=\frac{1}{2 g_{11}(r)}\left[\frac{\mathrm{d} g_{00}(r)}{\mathrm{d} r} \dot{t}^{2}-\frac{\mathrm{d} g_{11}(r)}{\mathrm{d} r} \dot{r}^{2}-2 r \dot{\phi}^{2}\right]$,

$\dot{\phi}=\frac{L}{r^{2}}$,

where $E$ and $L$ are the conserved energy and the angular momentum of the particle per-unit-mass, so $E$ is dimensionless and $L$ has units of mass, and the overdot stands for derivative with respect to the proper time, $\tau$. In terms of Cartesian coordinates, we denote the position and velocity components of the real orbit as $x, y, z$, and $v_{x}, v_{y}, v_{z}$. In our present case, $\theta=\pi / 2$, these are obtained using the transformation from spherical Schwarzschild coordinates to Cartesian coordinates:

$x=r \cos \phi$,

$y=r \sin \phi$,

$z=0$, 


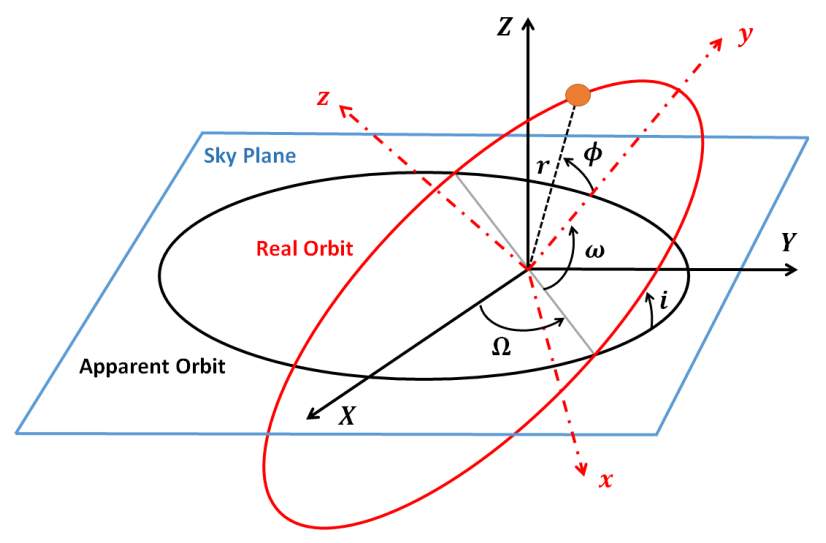

Fig. C.1. Projection of the real orbit onto the plane of the sky. The axes originate at Sgr A* (the focus of the ellipse). The picture illustrates the orbital parameters: $\phi$ is the azimuth angle of the spherical system of coordinates associated with the $x, y, z$ Cartesian coordinates, i.e., for an elliptic motion in the $x-y$ plane, it is the true anomaly, $i$ is the angle of inclination between the real orbit and the observation plane, $\Omega$ is the angle of the ascending node, and $\omega$ is the argument of pericenter. It is worth noting that the $Z$-axis of the coordinate system is defined by the vector pointing from the Solar System to the Galactic center.

and the corresponding three-velocities are

$v_{x}=v_{r} \cos \phi-r v_{\phi} \sin \phi$,

$v_{y}=v_{r} \sin \phi+r v_{\phi} \cos \phi$,

$v_{z}=0$

where $v_{r} \equiv u^{r} / u^{0}=\mathrm{d} r / \mathrm{d} t$ and $v_{\phi} \equiv u^{\phi} / u^{0}=\mathrm{d} \phi / \mathrm{d} t$, where $u^{\mu}=\mathrm{d} x^{\mu} / \mathrm{d} \tau$ is the four-velocity of the particle.

The solution of Eqs. (C.1a)-(C.1c) allows us to trace the stellar orbit, but to compare this with the observational data, it is necessary to determine the apparent orbit on the plane of the sky. Namely, we have to project the real orbit onto the observation plane, as shown in Fig. C.1. On the plane of the sky, the star traces an orbit with Cartesian positions $X_{\mathrm{obs}}$ and $Y_{\mathrm{obs}}$, defined by the observed angular positions, that is, the declination $\delta$ and the right ascension $\alpha$ (see, e.g., Ghez et al. 2008; Chu et al. 2018; Do et al. 2019):

$X_{\mathrm{obs}}=D_{\odot}\left(\alpha-\alpha_{\mathrm{SgrA} *}\right), \quad Y_{\mathrm{obs}}=D_{\odot}\left(\delta-\delta_{\mathrm{SgrA} *}\right)$,

centering the coordinate system on $\operatorname{Sgr} \mathrm{A}^{*}$. We adopted $D_{\odot}=$ 8 kpc (see, e.g., Gravity Collaboration 2018b; Do et al. 2019).

We introduce the same notation as in Do et al. (2019) for the classic Thiele-Innes constants, that is, $A, B, C, F, G$, and $H$. The theoretical apparent orbit (i.e., the position in coordinates $X, Y$, and $Z$ ) can then be obtained from the real orbit positions $x$ and $y$ by (see Fig. C.1)

$X=x B+y G$

$Y=x A+y F$,

$Z=x C+y H$,

and the corresponding components of the apparent coordinate velocity are

$V_{X}=\frac{\mathrm{d} X}{\mathrm{~d} t}=v_{x} B+v_{y} G$,

$V_{Y}=\frac{\mathrm{d} Y}{\mathrm{~d} t}=v_{x} A+v_{y} F$

$V_{Z}=\frac{\mathrm{d} Z}{\mathrm{~d} t}=v_{x} C+v_{y} H$, where

$A=\cos \Omega \cos \omega-\sin \Omega \sin \omega \cos i$,

$B=\sin \Omega \cos \omega+\cos \Omega \sin \omega \cos i$,

$C=\sin \omega \sin i$,

$F=-\cos \Omega \sin \omega-\sin \Omega \cos \omega \cos i$,

$G=-\sin \Omega \sin \omega+\cos \Omega \cos \omega \cos i$,

$H=\cos \omega \sin i$,

where $\omega, i$, and $\Omega$ are the osculating orbital elements: the argument of pericenter, the inclination between the real orbit and the observation plane, and the ascending node angle, respectively. These orbital elements are strictly defined (fixed constants) only for a Keplerian (Newtonian) elliptic orbit. In this case, the radial position is simply given by $r=a(1-e \cos E)$, where $a$ is the semimajor axis of the ellipse, $e$ its eccentricity, and $E$ is its eccentric anomaly. The latter is related to the true anomaly, which is the azimuthal angle $\phi$, by $\cos \phi=(\cos E-e) /$ $(1-e \cos E)$. In this case, Eqs. (C.9) and (C.10) reduces to Eqs. (S8)-(S10) of Do et al. (2019). However, in the full general relativistic case, it is not possible (in general) to determine a closed form in which an analytic function $r(\phi)$ describes the orbit. For the simpler case of a test particle that moves around a Schwarzschild BH, $r(\phi)$ can be written in terms of Jacobi elliptic functions. In the RAR model, we obtain $r(\tau)$ and $\phi(\tau)$, or for the sake of comparison with observations, $r(t)$ and $\phi(t)$, by numerical integration of the equations of motion, Eq. (C.1a). Clearly, we can then obtain $r(\phi)$ numerically.

\section{C.2. Fitting procedure of the observational data}

To fit the observed positions, Do et al. (2019) introduced timevarying offsets of the position of the gravitational center of mass with respect to the center of the reference frame, adopting a linear drift. For our purpose, it is sufficient to introduce the constant offsets $X_{0}$ and $Y_{0}$,

$X_{\mathrm{obs}}\left(t_{\mathrm{obs}}\right)=X[r(t), \phi(t) ; \omega, i, \Omega]+X_{0}$,

$Y_{\mathrm{obs}}\left(t_{\mathrm{obs}}\right)=Y[r(t), \phi(t) ; \omega, i, \Omega]+Y_{0}$,

where $X$ and $Y$ are given by Eq. (C.9), $t_{\mathrm{obs}}$ is the time measured at the observer point, and $t=t_{\mathrm{em}}$ is the time at emission.

In general, $t_{\mathrm{obs}}$ and $t$ are not equal: a time delay exists in the observations that is caused by light-propagation effects along the line of sight. An obvious cause of the time delay is the fact that the speed of light is finite. Along the line of sight (i.e., the Zdirection), this is called Rømer delay (see, e.g., Do et al. 2019),

$t_{\mathrm{obs}}=t_{\mathrm{em}}+\frac{Z\left(t_{\mathrm{em}}\right)}{c}$,

where $Z$ is given by Eq. (C.9c). Equation (C.13) is an implicit nonlinear equation for $t_{\mathrm{em}}$, but it can be inverted at first order as (see, e.g., Do et al. 2019)

$t_{\mathrm{em}} \approx t_{\mathrm{obs}}-\frac{Z\left(t_{\mathrm{obs}}\right)}{c}$.

We neglected any photon delay time in our fitting procedure, therefore we adopted

$t_{\mathrm{em}}=t_{\mathrm{obs}}$,

which is sufficiently accurate for the purposes of this work. The model parameters we inferred (see Table 1) of S2 in the 


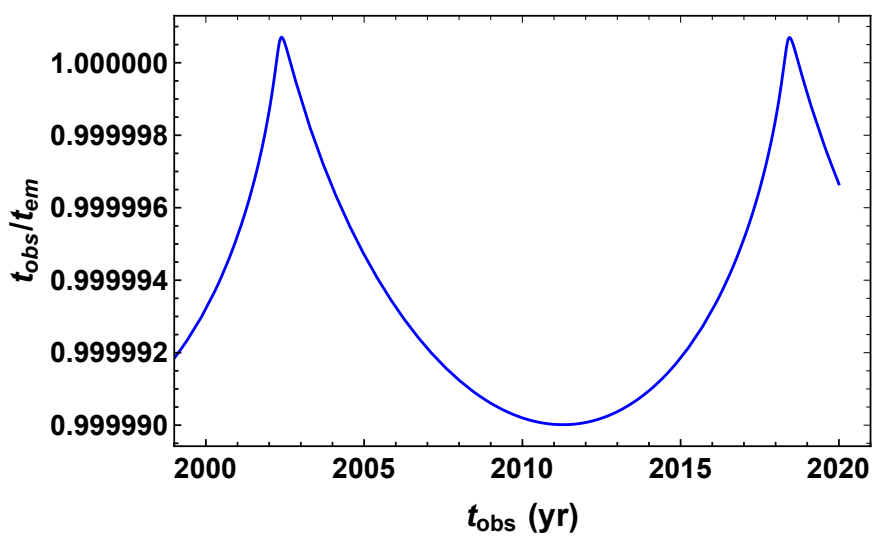

Fig. C.2. Ratio $t_{\mathrm{obs}} / t_{\mathrm{em}}$ as given by Eq. (C.14), calculating $Z\left(t_{\mathrm{obs}}\right)$ with Eq. (C.9c), for the best-fit model parameters of the BH model Table 1, derived assuming Eq. (C.15).

case of a Schwarzschild $\mathrm{BH}$ are similar to those that were previously presented in the literature, see, for instance, Gravity Collaboration (2018b) and Do et al. (2019) for a comparison. Figure C.2 shows that $t_{\mathrm{obs}} \approx t_{\mathrm{em}}$ with high accuracy $(\approx 0.001 \%$ error $)$. Our estimate shown in Fig. C. 2 agrees with the estimate in Do et al. (2019), who reported that this delay modulates the light-propagation time by $\Delta t=t_{\mathrm{obs}}-t_{\mathrm{em}} \approx-0.5$ days at pericenter and $\Delta t \approx 7.5$ days at apocenter.

The assumption of zero relative motion of the center of mass and the center of the observer's frame introduces only a difference of order $v_{z 0} / v_{Z} \sim 0.1 \%$ in the radial velocity, being $z$ the redshift function (see Appendix B for details).

In general, the four-velocity component $u_{Z}$ is not directly accessible from the observations as it is the redshift function $z$ given by Eq. (B.5). Therefore we obtained the parameters that best fit the equation

$z_{\mathrm{obs}}\left(t_{\mathrm{obs}}\right)=z[r(t), \phi(t), \dot{r}(t), \dot{\phi}(t) ; \omega, i]$,

where in terms of the orbital parameters,

$z=\gamma-1+u_{Z}$

$u_{Z}=\gamma V_{Z}=[\dot{r} \sin (\phi+\omega)+r \dot{\phi} \cos (\phi+\omega)] \sin i$.

Here we introduced the notation $V_{Z} \equiv \boldsymbol{v} \cdot \boldsymbol{n}$, where $\boldsymbol{n}$ is the unit vector pointing from the emitter to the observer (i.e., the unit vector in the direction of the line of sight), and we recall that $\dot{r}=\mathrm{d} r / \mathrm{d} \tau, \dot{\phi}=\mathrm{d} \phi / \mathrm{d} \tau$, and $\gamma$ is given by Eq. (B.3).

It is now clear that at every time, the possible available observational data are the coordinates of the apparent orbit in the sky plane, that is, $X_{\mathrm{obs}}$ and $Y_{\mathrm{obs}}$, and the measured redshift function, $z_{\text {obs }}$. The real orbit at every time is obtained by solving the equations of motion, Eq. (C.1a), which give the coordinate positions $r(t), \phi(t)$, and the corresponding velocities $\dot{r}(t)$ and $\dot{\phi}(t)$.

First, to solve Eq. (C.1a), we must set the value of $E$ and $L$. From the definition of the Lorentz factor, Eq. (B.3), and the equation of motion for $t(\tau)$, Eq. (C.1a), we obtain the first integral:

$-g_{00}(r) g_{11}(r) \dot{r}^{2}=E^{2}-U_{\mathrm{eff}}^{2}(r)$,

where

$U_{\mathrm{eff}}^{2}(r) \equiv g_{00}(r)\left(1+\frac{L^{2}}{r^{2}}\right)$, is the well-known effective potential governing the radial motion. The relevance of this equation is that it allows us to perform a turning-point analysis, analogously to the classical Kepler problem. Equation (C.18) shows that the request of having a bound, closed orbit within two known turning points, that is, the pericenter $\left(r_{\mathrm{p}}\right)$ and the apocenter $\left(r_{a}\right)$, where $\dot{r}=0$, implies a unique solution for $E$ and $L$; see Fig. C.3. The value of $U_{\mathrm{eff}}$ at the turning points has to be the same, so that we obtain $L$ by solving the algebraic equation

$U_{\text {eff }}\left(L, r_{p}\right)=U_{\text {eff }}\left(L, r_{a}\right)$,

and with the knowledge of $L$, we obtain the energy by

$E=U_{\mathrm{eff}}\left(L, r_{p}\right), \quad$ or $\quad E=U_{\mathrm{eff}}\left(L, r_{a}\right)$

The metric functions $g_{00}(r)$ and $g_{11}(r)$ in the BH case are set by the mass of the $\mathrm{BH}, M_{\mathrm{BH}}$. In the extended RAR model, the parameters $\theta_{0}, \beta_{0}, W_{0}$, and the fermion mass $m$ are well constrained by the rotation curves of the Galaxy (see Appendix A and Argüelles et al. 2018, 2019a, for details). Each possible set of parameters gives a mass of the quantum core, $M_{\mathrm{c}}$ (or alternatively, of the central density; see Argüelles et al. 2019a for details), therefore the metric functions are known when we chose a value of $M_{\mathrm{c}}$ for given halo boundary conditions in agreement with observables (see Appendix A).

After setting the metric functions (i.e., given $M_{\mathrm{c}}$ in the extended-RAR model or $M_{\mathrm{BH}}$ in the $\mathrm{BH}$ model), calculating the values of $E$ and $L$ with given pericenter $r_{\mathrm{p}}$ and apocenter $r_{\mathrm{a}}$ distances (or alternatively, the semimajor axis $a$ and the eccentricity $e$ ), we can integrate the equations of motion (C.1a)-(C.1c) giving appropriate initial conditions at initial proper time $\tau_{0}$. We give them at the apocenter, that is, we set $t_{0} \equiv t\left(\tau_{0}\right)=0, r_{0} \equiv r\left(t_{0}\right)=$ $r_{a}, \phi_{0} \equiv \phi\left(t_{0}\right)=\pi$, and $\dot{r}\left(t_{0}\right)=0$. We numerically integrate the equations of motion through an adaptive integrator based on the fourth-order Runge-Kutta (RKF45) method (Fehlberg 1970). We thus obtain $t(\tau), r(t)=r[\tau(t)], \phi(t)=\phi[\tau(t)]$. We recall that $t$ is the coordinate time at emission point, which is within our adopted approximation of zero time-delay of the photons; see Eq. (C.15).

When the variables of the dynamics of the real orbit were calculated, we proceeded to obtain the orbital elements $i, \omega, \Omega$, and the constant offsets $X_{0}$ and $Y_{0}$ from the request that the predicted orbit, that is, $X(t)$ and $Y(t)$, Eq. (C.9), and the predicted redshift function $z$ fit the observational values, that is, $X_{\mathrm{obs}}, Y_{\mathrm{obs}}$, and $z_{\mathrm{obs}}$, respectively.

In order to quantify the goodness of fit, we computed the reduced $-\chi^{2}$ for each of the observables,

$$
\begin{aligned}
& \bar{\chi}_{X}^{2}=\frac{1}{N_{X}-p} \sum_{j=1}^{N_{X}} \frac{\left[X_{\mathrm{obs}, j}-\left(X+X_{0}\right)\right]^{2}}{\Delta X_{\mathrm{obs}, j}^{2}}, \\
& \bar{\chi}_{Y}^{2}=\frac{1}{N_{Y}-p} \sum_{j=1}^{N_{Y}} \frac{\left[Y_{\mathrm{obs}, j}-\left(Y+Y_{0}\right)\right]^{2}}{\Delta Y_{\mathrm{obs}, j}^{2}}, \\
& \bar{\chi}_{z}^{2}=\frac{1}{N_{z}-p} \sum_{j=1}^{N_{z}} \frac{\left(z_{\mathrm{obs}, j}-z\right)^{2}}{\Delta z_{\mathrm{obs}, j}^{2}},
\end{aligned}
$$

where the subscript $j$ indicates the $j$ th data element of the observable $\left\{X_{\mathrm{obs}, j}, Y_{\mathrm{obs}, j}, z_{\mathrm{obs}, j}\right\},\left\{\Delta X_{\mathrm{obs}, j}, \Delta Y_{\mathrm{obs}, j}, \Delta z_{\mathrm{obs}, j}\right\}$ is the associated standard deviation of the $j$-th measurement, $\left\{N_{X}, N_{Y}, N_{z}\right\}$ are the number of data elements of the observable, and $p$ is the number of model parameters. 

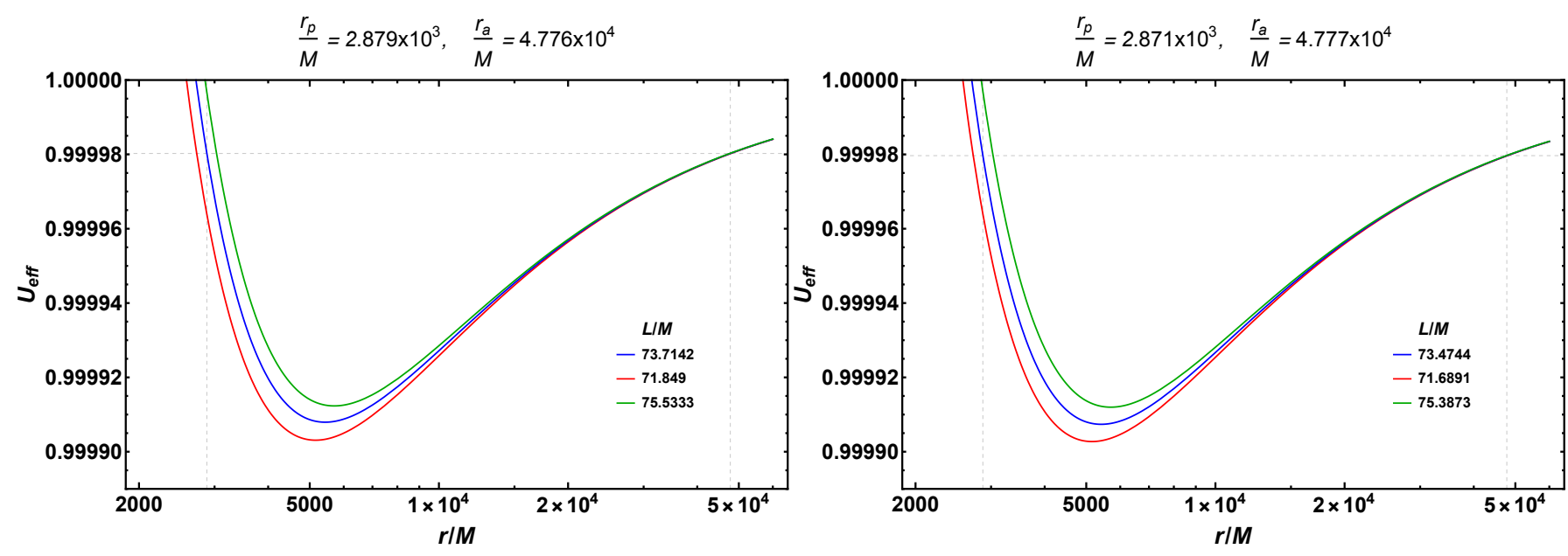

Fig. C.3. Effective potential $U_{\text {eff }}$ given by Eq. (C.19) for selected values of the conserved angular momentum L. Left: massive BH case, i.e., Schwarzschild solution, $g_{00}=1-2 M / r$. Right: DM case; $g_{00}$ obtained from numerical integration of the general relativistic equilibrium equations of the extended RAR model for a fermion mass $m c^{2}=56 \mathrm{keV}$, see Appendix A for details. Imposing a bound orbit within given values of the pericenter and apocenter (vertical dashed lines), in this example, $r_{\mathrm{p}} / M=2.976 \times 10^{3}$ and $r_{\mathrm{a}} / M=4.714 \times 10^{4}$, respectively, implies a unique solution of $E$ (dashed horizontal value) and $L$ (value associated with the blue curve). In this example, the adopted mass of the massive BH for the Schwarzschild solution is $M_{\mathrm{BH}} \equiv M=4.075 \times 10^{6} M_{\odot}$, and for the mass of the DM RAR core, it was set to $M_{\mathrm{c}}=3.5 \times 10^{6} M_{\odot}$.

For the best match with the observational data at the observational times, which are presented in J2000 convention, we performed a time shift of the theoretical data, $\Delta t$. To do this, we introduce the new time $t^{\prime} \equiv t-\Delta t$, that is, we must calculate $r\left(t^{\prime}\right)=r(t-\Delta t), \phi\left(t^{\prime}\right)=\phi(t-\Delta t)$, etc. Thus, the time shift $\Delta t$ becomes one of the parameters of the fitting process. With this, Eqs. (C.12a)-(C.12b) and Eqs. (C.16) are solved in iterative fashion by varying $\Delta t$ and calculating the orbital parameters that minimize $\bar{\chi}_{X}^{2}, \bar{\chi}_{Y}^{2}$, and $\bar{\chi}_{z}^{2}$ for each value of $\Delta t$. In general, we find that the fit of the redshift function is better than the position fits. This holds for S2 and G2 because the observational data of the position are somewhat scattered at times. In any case, in addition to the individual $\chi^{2}$ values, we evaluated the overall performance of every set of parameters by computing the mean of the $\chi^{2}$,

$\left\langle\chi^{2}\right\rangle \equiv \frac{1}{3}\left(\bar{\chi}_{X}^{2}+\bar{\chi}_{Y}^{2}+\bar{\chi}_{z}^{2}\right)$

The values of the model parameters reported in Table 1 correspond to those that generate the smallest mean $\left\langle\chi^{2}\right\rangle$ for the range of parameters explored. We also report the individual $\bar{\chi}_{X}^{2}, \bar{\chi}_{Y}^{2}$, and $\bar{\chi}_{z}^{2}$. It is important to note that for different values of the parameters we were able to obtain a better fit of a specific single observable, for example, $z_{\mathrm{obs}}$. For instance, we found some set of parameters for S2 that yield for $\bar{\chi}_{z}^{2}$ a value as low as 1.03 , with respect to the value $\bar{\chi}_{z}^{2} \approx 1.28$ of the set of parameters that leads to the smallest $\left\langle\chi^{2}\right\rangle$ (see Table 1).

To summarize, our fitting procedure for a given core mass $M_{\mathrm{c}}$ of the RAR model or for a BH mass $M_{\mathrm{BH}}$ in the massive $\mathrm{BH}$ model performs the following steps:

1. Set a value for the eccentricity $e$.

2. Set a value for the semimajor axis $a$.

3. Calculate the pericenter $r_{p}$ and apocenter $r_{\mathrm{a}}$ for the chosen $e$ and $a$.
4. Using Eqs. (C.20) and (C.21), calculate $L$ and $E$ to integrate the equations of motion (C.1a) $-(\mathrm{C} .1 \mathrm{c})$ with initial conditions at apocenter: $t_{0}=0, r_{0}=r_{a}, \phi_{0}=\pi$ and $\dot{r}\left(t_{0}\right)=0$;

5. Set a value for the constant time shift $\Delta t$;

6. Calculate all quantities of the real orbit at the shifted time $t^{\prime}=t-\Delta t$, that is, $r\left(t^{\prime}\right), \phi\left(t^{\prime}\right), \dot{r}\left(t^{\prime}\right)$, and $\dot{\phi}\left(t^{\prime}\right)$.

7. At this stage, the redshift function depends only on the orbital elements $\omega$ and $i$, see Eq. (C.16), so that we obtain them by minimizing $\bar{\chi}_{z}^{2}$, Eq. (C.22c).

8. We iterate the above steps 5-7 in an appropriate range of $\Delta t$, calculate the sets $\{\Delta t, \omega, i\}$ that lead to each minimum $\bar{\chi}_{z}^{2}$, and identify the set leading to the infimum $\bar{\chi}_{z}^{2}$, that is, the smallest $\bar{\chi}_{z}^{2}$.

9. Set a value of $\Omega$.

10. At this stage, the $X$ position only depends on the offset $X_{0}$, see Eq. (C.12a), so that we obtain it by minimizing $\bar{\chi}_{X}^{2}$, Eq. (C.22a).

11. Likewise, the $Y$ position only depends on the offset $Y_{0}$, see Eq. (C.12b), so that we obtain it by minimizing $\bar{\chi}_{Y}^{2}$, Eq. (C.22b).

12. We iterate the above steps $9-11$ in an appropriate range of $\Omega$, calculate the sets $\left\{\Omega, X_{0}, Y_{0}\right\}$ that lead to each minimum of $\bar{\chi}_{X}^{2}$ and $\bar{\chi}_{Y}^{2}$, and identify the set leading to the infimum of $\bar{\chi}_{X}^{2}$ and of $\bar{\chi}_{Y}^{2}$, that is, the smallest $\bar{\chi}_{X}^{2}$ and $\bar{\chi}_{Y}^{2}$.

13. Based on the lowest values of $\bar{\chi}_{X}^{2}, \bar{\chi}_{Y}^{2}$, and $\bar{\chi}_{z}^{2}$, calculate the mean $\left\langle\chi^{2}\right\rangle$ given by Eq. (C.23).

14. Steps $1-13$ are iterated for different values of $e$ and $a$ in some appropriate range.

15. Identify the best-fit parameters as those that lead to the smallest $\left\langle\chi^{2}\right\rangle$.

16. Steps $1-15$ can be repeated for different values of the mass of the DM core $M_{\mathrm{c}}$ in the extended RAR model, or of the $\mathrm{BH}$ mass $M_{\mathrm{BH}}$ in the central massive $\mathrm{BH}$ model. 